\title{
EXISTENCE AND BLOW-UP BEHAVIOR FOR SOLUTIONS OF THE GENERALIZED JANG EQUATION
}

\author{
QING HAN AND MARCUS KHURI
}

\begin{abstract}
The generalized Jang equation was introduced in an attempt to prove the Penrose inequality in the setting of general initial data for the Einstein equations. In this paper we give an extensive study of this equation, proving existence, regularity, and blow-up results. In particular, precise asymptotics for the blow-up behavior are given, and it is shown that blow-up solutions are not unique.
\end{abstract}

\section{INTRODUCTION}

In 1978 the physicist P.-S. Jang [14] introduced a quasilinear elliptic equation in connection with the positive energy conjecture of General Relativity. Since then it has had numerous applications from quasilocal mass to the existence of black holes. An excellent survey of these applications may be found in [3]. However, Jang's equation has been shown to be unsuitable for an application to the Penrose inequality [16], and for this reason a generalization of this equation has been proposed in [5] and [6]. Further applications may be found in [7] and [15]. The purpose of this paper is to give a complete analysis of the generalized Jang equation, in a setting suitable for the Penrose inequality.

Consider an initial data set $(M, g, k)$ for the Einstein equations. This consists of a Riemannian 3 -manifold $M$ with metric $g$, and a symmetric 2 -tensor $k$, which satisfy the constraint equations:

$$
\begin{aligned}
2 \mu & =R+(\operatorname{Trk})^{2}-|k|^{2}, \\
J & =\operatorname{div}(k+(\operatorname{Trk}) g) .
\end{aligned}
$$

Here $\mu$ and $J$ are the energy and momentum densities of the matter fields, respectively, and $R$ is the scalar curvature of $g$. If all measured energy densities are nonnegative then $\mu \geq|J|$, which will be referred to as the dominant energy condition. This condition may be viewed as a quasi nonnegativity of the scalar curvature. In fact in the time symmetric $(k=0)$ or maximal case $(\operatorname{Tr} k=0)$, one does have $R \geq 0$, and this condition plays a central role in the proof of several results such as the positive energy theorem [18], 20] and the Penrose inequality [4], 13]. Moreover, the primary difficulty in establishing these results for general initial data is the lack of nonnegative scalar curvature. Thus, in the general case, one is motivated to deform the initial data in an appropriate way (depending on the problem at hand) such that the dominant energy condition yields nonnegativity of the scalar curvature for the deformed metric. The deformation chosen by Jang [14] is given by $\bar{g}=g+d f^{2}$, for some function $f$ defined on $M$. Notice that $\bar{g}$ is the induced metric on the graph $\Sigma=\{t=f(x)\}$ in the product 4-manifold $\left(M \times \mathbb{R}, g+d t^{2}\right)$. When viewed in this way, the optimal Jang deformation is given by a hypersurface $\Sigma \subset\left(M \times \mathbb{R}, g+d t^{2}\right)$ which has scalar curvature that is as nonnegative as possible. It turns out that this optimal deformation arises as the hypersurface which satisfies Jang's

The first author acknowledges the support of NSF Grant DMS-1105321. The second author acknowledges the support of NSF Grant DMS-1007156 and a Sloan Research Fellowship. MSC Codes: 35Q75, 35J70, 83C99, 58J32. 
equation

$$
H_{\Sigma}-\operatorname{Tr}_{\Sigma} k=0
$$

where $H_{\Sigma}$ is mean curvature and $\operatorname{Tr}_{\Sigma} k$ denotes the trace of $k$ (extended trivially to the 4-manifold) over $\Sigma$. While this deformation does not necessarily yield nonnegative scalar curvature, it is sufficient to make a further conformal deformation to zero scalar curvature, as was carried out by Schoen and Yau [19] in their proof of the positive energy theorem. Jang's approach also beautifully handles the case of equality for the positive energy theorem. In the case of equality, the Jang surface $(\Sigma, \bar{g})$ is isometric to Euclidean space $\left(\mathbb{R}^{3}, \delta\right)$, and hence $g=\delta-d f^{2}$. It follows that the map $x \mapsto(x, f(x))$ yields an isometric embedding of $(M, g)$ into the Minkowski spacetime $\mathbb{M}^{4}$; it can also be shown that the second fundamental form of this embedding agrees with $k$, as desired. In some sense, Jang's procedure seems to be tailor made for the positive energy theorem. In fact it is so well calibrated to the positive energy theorem, that this method is rendered inapplicable to the Penrose inequality. The reason for this is as follows. As explained in [5] and [6], in the case of equality for the Penrose inequality, the Jang metric $\bar{g}$ should coincide with $g_{s c}$, the induced metric on the $t=0$ slice of the Schwarzschild spacetime $\mathbb{S C}^{4}$. It follows that $g=g_{s c}-d f^{2}$, and the map $x \mapsto(x, f(x))$ cannot yield an embedding of $(M, g)$ into $\mathbb{S C}^{4}$, since the Schwarzschild metric has the warped product structure $-\phi_{s c}^{2} d t^{2}+g_{s c}$. However, this observation leads to a natural modification of the Jang approach from which the generalized Jang equation arises.

We now search for a hypersurface $\Sigma$, given by a graph $t=f(x)$, inside the warped product space $\left(M \times \mathbb{R}, g+\phi^{2} d t^{2}\right)$, where $\phi$ is a nonnegative function defined on $M$. For certain applications the choice of $\phi$ may depend on $f$, however in this paper we will assume that $\phi$ is fixed independent of $f$. The goal or motive which leads to the generalized Jang equation is the same as in the classical case. That is, we search for a hypersurface which has the most positive scalar curvature that is possible. In order to have any chance of obtaining a positivity property for the scalar curvature, we would like the Jang surface $\Sigma$ to satisfy an equation with the same structure as in (1.2), namely

$$
H_{\Sigma}-\operatorname{Tr}_{\Sigma} K=0
$$

where again $H_{\Sigma}$ is mean curvature and $\operatorname{Tr}_{\Sigma} K$ denotes the trace of $K$ over $\Sigma$. Here, however, $K$ represents a nontrivial extension of the initial data $k$ to all of $M \times \mathbb{R}$. In (1.2) $k$ is extended trivially in that $k\left(\partial_{t}, \cdot\right)=0$, but it turns out that the trivial extension is not appropriate for a Jang surface inside the warped product metric. This is due to the fact that for applications it is desirable for solutions of the generalized Jang equation to blow-up at apparent horizons, and as is shown in [5], this is possible when $K$ is extended as follows:

$$
\begin{array}{rlrl}
K\left(\partial_{x^{i}}, \partial_{x^{j}}\right)=K\left(\partial_{x^{j}}, \partial_{x^{i}}\right) & =k\left(\partial_{x^{i}}, \partial_{x^{j}}\right) & \text { for } \quad & 1 \leq i, j \leq 3, \\
K\left(\partial_{x^{i}}, \partial_{t}\right)=K\left(\partial_{t}, \partial_{x^{i}}\right) & =0 \quad \text { for } \quad 1 \leq i \leq 3, \\
K\left(\partial_{t}, \partial_{t}\right) & =\frac{\phi^{2} g(\nabla f, \nabla \phi)}{\sqrt{1+\phi^{2}|\nabla f|^{2}}} &
\end{array}
$$

where $x^{i}, i=1,2,3$, are local coordinates on $M$. Moreover this particular extension also yields an optimal positivity property for the scalar curvature of solutions to (1.3). Namely, a long calculation ([5], 6]) gives the following formula for the scalar curvature of $\Sigma$ satisfying equation (1.3) with extension (1.4):

$$
\bar{R}=2(\mu-J(w))+\left.|A-K|_{\Sigma}\right|^{2}+2|q|^{2}-2 \phi^{-1} \overline{\operatorname{div}}(\phi q) .
$$


Here $\bar{g}=g+\phi^{2} d f^{2}$ and $A$ are the induced metric and second fundamental form of $\Sigma$, respectively, $\left.K\right|_{\Sigma}$ is the restriction to $\Sigma$ of the extended tensor $K, \overline{d i v}$ is the divergence operator with respect to $\bar{g}$, and $q$ and $w$ are 1 -forms given by

$$
w_{i}=\frac{\phi f_{i}}{\sqrt{1+\phi^{2}|\nabla f|^{2}}}, \quad q_{i}=\frac{\phi f^{j}}{\sqrt{1+\phi^{2}|\nabla f|^{2}}}\left(A_{i j}-\left(\left.K\right|_{\Sigma}\right)_{i j}\right),
$$

with $f^{j}=g^{i j} f_{i}$. If the dominant energy condition is satisfied, then all terms appearing on the right-hand side of (1.5) are nonnegative, except possibly the last term. However the last term has a special structure, and in many applications it is clear that a specific choice of $\phi$ will allow one to 'integrate away' this divergence term, so that in effect the scalar curvature is weakly nonnegative (that is, nonnegative when integrated against certain functions).

When the tensor $k$ is extended according to (1.4), we will refer to equation (1.3) as the generalized Jang equation, and the solution $\Sigma=\{t=f(x)\}$ will be called the Jang surface. In local coordinates the generalized Jang equation takes the following form:

$$
\left(g^{i j}-\frac{\phi^{2} f^{i} f^{j}}{1+\phi^{2}|\nabla f|^{2}}\right)\left(\frac{\phi \nabla_{i j} f+\phi_{i} f_{j}+\phi_{j} f_{i}}{\sqrt{1+\phi^{2}|\nabla f|^{2}}}-k_{i j}\right)=0 .
$$

This is a quasilinear elliptic equation, which degenerates when $f$ blows-up or $\phi=0$. When $\phi=1$ this reduces to the classical Jang equation studied by Schoen and Yau [19].

Our study of (1.6) is naturally divided into two steps. In the first step, the setting consists of a complete initial data set on which the function $\phi$ is strictly positive. We will show that an analogue of the existence and regularity result, as obtained by Schoen and Yau [19] for the classical Jang equation, holds in this case. The proof follows the same general ideas present in [19] with appropriate modification. The primary difference is that (especially for applications) it is important to track precisely how the estimates depend on $\phi$ and its derivatives. These estimates will also be helpful for the case in which $\phi$ is allowed to vanish. In the second and primary step, the setting consists of an initial data set with outermost apparent horizon boundary along which $\phi$ vanishes. Our study in this case constitutes the main focus and purpose of this paper. We will establish the existence of solutions which blow up along the boundary, and will give precise descriptions of the blow-up rates by constructing upper and lower barriers. The fact that $\phi$ vanishes on the boundary adds an extra degeneracy to equation (1.6), which makes the analysis in this case much more difficult. The existence of blow-up solutions for the classical Jang equation has been proven by Metzger in [17] (see also [8]).

In this paper we will always assume that the initial data are asymptotically flat (with one end), so that at spatial infinity the metric and extrinsic curvature satisfy the following fall-off conditions

$$
\left|\partial^{m}\left(g_{i j}-\delta_{i j}\right)\right|=O\left(|x|^{-m-1}\right), \quad\left|\partial^{m} k_{i j}\right|=O\left(|x|^{-m-2}\right), \quad m=0,1,2, \quad \text { as } \quad|x| \rightarrow \infty .
$$

Moreover if the initial data has boundary, it will be assumed to consist of an outermost apparent horizon. To explain this more precisely, recall that the strength of the gravitational field in the vicinity of a 2-surface $S \subset M$ may be measured by the null expansions

$$
\theta_{ \pm}:=H_{S} \pm T r_{S} k
$$

where $H_{S}$ is the mean curvature with respect to the unit outward normal (pointing towards spatial infinity). The null expansions measure the rate of change of area for a shell of light emitted by the surface in the outward future direction $\left(\theta_{+}\right)$, and outward past direction $\left(\theta_{-}\right)$. Thus the gravitational field is interpreted as being strong near $S$ if $\theta_{+}<0$ or $\theta_{-}<0$, in which case $S$ is referred to as a future 
(past) trapped surface. Future (past) apparent horizons arise as boundaries of future (past) trapped regions and satisfy the equation $\theta_{+}=0\left(\theta_{-}=0\right)$. In the setting of the initial data set formulation of the Penrose inequality, apparent horizons take the place of event horizons, in that the area of the event horizon is replaced by the least area required to enclose the outermost apparent horizon. Here, an outermost future (past) apparent horizon refers to a future (past) apparent horizon outside of which there is no other apparent horizon; such a horizon may have several components, each having spherical topology $([9],[10])$. In this paper we will refer to the union of the outermost future apparent horizon and outermost past apparent horizon as the outermost apparent horizon, and we will assume that past and future horizon components do not intersect. For the sake of applications it is desirable for solutions of the generalized Jang equation to blow-up to $+\infty(-\infty)$ in the form of a cylinder over the future (past) components of an outermost apparent horizon, and to vanish at spatial infinity. The fall-off rate for the solution of the generalized Jang equation depends on the asymptotics of the warping factor, however here we will always assume that

$$
\phi(x)=1+\frac{C}{|x|}+O\left(\frac{1}{|x|^{2}}\right) \quad \text { as } \quad|x| \rightarrow \infty
$$

for some constant $C$, which yields

$$
\left|\nabla^{m} f\right|(x)=O\left(|x|^{-\frac{1}{2}-m}\right) \quad \text { as } \quad|x| \rightarrow \infty, \quad m=0,1,2 .
$$

These asymptotics ensure that the ADM energy of the Jang surface agrees with that of the initial data.

As mentioned above, our primary goal is to study (1.6) in the setting of initial data with outermost horizon boundary along which $\phi$ vanishes, and to describe the blow-up behavior of its solutions near the boundary. To this end, we will need to know the asymptotics of the warping factor $\phi$ and of the null expansions. That is, we assume that the warping factor and the null expansions vanish in a controlled way at the horizon. Set $\tau(x)=\operatorname{dist}(x, \partial M)$. In a neighborhood of $\partial M$, we impose the following structure condition

$$
\phi(x)=\tau^{b}(x) \widetilde{\phi}(x),
$$

for some smooth (up to the boundary) strictly positive function $\widetilde{\phi}$, with $b \geq 0$. Next, we let $S_{\tau}$ denote level sets of the geodesic flow emanating from $\partial M$, that is, each point of $S_{\tau}$ is of distance $\tau$ from the boundary. Furthermore decompose $\partial M=\partial_{+} M \cup \partial_{-} M$, where $\partial_{+} M\left(\partial_{-} M\right)$ denotes the future (past) apparent horizon components. We then stipulate that near $\partial_{ \pm} M$

$$
\left|\theta_{ \pm}\left(S_{\tau}\right)\right| \leq c \tau^{l}
$$

for some constants $l, c>0$. Notice that when the initial data are smooth up to the boundary, as will always be the case in this paper, $l \geq 1$. Whether or not $f$ actually blows up and approximates a cylinder over the horizon is highly dependent on the relationship between the vanishing rates of $\theta_{ \pm}$ and $\phi$, that is, the relation between $l$ and $b$. In some cases we require a more restrictive condition

$$
c^{-1} \tau^{l} \leq \theta_{ \pm}\left(S_{\tau}\right) \leq c \tau^{l},
$$

with constants $l, c>0$.

The main result in this paper is the following theorem.

Theorem 1.1. Suppose that $(M, g, k)$ is a smooth, asymptotically flat initial data set, with outermost apparent horizon boundary $\partial M=\partial_{+} M \cup \partial_{-} M$. Suppose further that $\phi$ is smooth, strictly positive away from $\partial M$, and satisfies (1.8) and (1.10) for some $b \geq 0$. 
(1) If $-\frac{l-1}{2} \leq b<\frac{l+1}{2}$ and (1.12) is valid for some $l \geq 1$, then there exists a smooth solution $f$ of the generalized Jang equation (1.6), satisfying (1.9), and such that $f(x) \rightarrow \pm \infty$ as $x \rightarrow \partial_{ \pm} M$. More precisely, in a neighborhood of $\partial_{ \pm} M$

$$
\begin{gathered}
\alpha^{-1} \tau^{-b-\frac{l-1}{2}}+\beta^{-1} \leq \pm f \leq \alpha \tau^{-b-\frac{l-1}{2}}+\beta \quad \text { if } \quad-\frac{l-1}{2}<b<\frac{l+1}{2} \\
-\alpha^{-1} \log \tau+\beta^{-1} \leq \pm f \leq-\alpha \log \tau+\beta \quad \text { if } \quad b=-\frac{l-1}{2}
\end{gathered}
$$

for some positive constants $\alpha$ and $\beta$.

(2) If $\frac{1}{2} \leq b<\frac{l+1}{2}$ and (1.11) is valid for some $l \geq 1$, then there exists a smooth solution $f$ of the generalized Jang equation (1.6), satisfying (1.9), and such that $f(x) \rightarrow \pm \infty$ as $x \rightarrow \partial_{ \pm} M$. More precisely, in a neighborhood of $\partial_{ \pm} M$

$$
\begin{gathered}
\alpha^{-1} \tau^{1-2 b}+\beta^{-1} \leq \pm f \leq \alpha \tau^{1-2 b}+\beta \quad \text { if } \quad \frac{1}{2}<b<\frac{l+1}{2}, \\
-\alpha^{-1} \log \tau+\beta^{-1} \leq \pm f \leq-\alpha \log \tau+\beta \quad \text { if } \quad b=\frac{1}{2},
\end{gathered}
$$

for some positive constants $\alpha$ and $\beta$.

Remark 1.2. The hypothesis $b \geq-\frac{l-1}{2}$ is trivially satisfied under the primary assumptions of this paper, that is $b \geq 0$ and $l \geq 1$. However it is believed that this theorem, and others having this hypothesis, continue to hold even when these primary assumptions are relaxed. For this reason, the inequality $b \geq-\frac{l-1}{2}$ is included in the statement of such results.

We note that there are at least two solutions with different asymptotics when $\frac{1}{2} \leq b<\frac{l+1}{2}$. The estimates (1.13) and (1.14) are established by constructing appropriate sub and super solutions, however these estimates may not hold when the particular inequalities between $l$ and $b$ are not satisfied. Furthermore, we exhibit by example, a solution which does not have cylindrical asymptotics near the outermost apparent horizon when $b=(l+1) / 2$.

It should be pointed out that the lower bounds in (1.13) are new even for the classical Jang equation, that is, when $\phi=1$.

Theorem 1.1 establishes the existence of solutions to the generalized Jang equation which possess appropriate behavior for application to the Penrose inequality. In particular, for an appropriate choice of $\phi$, the blow-up rates (1.14) show that the Jang surface is a manifold with boundary, and that the boundary is a minimal surface. This allows techniques from the time symmetric proof of the Penrose inequality to be applied, and is different from the behavior of the blow-up solutions of the classical Jang equation which possess an infinitely long neck at the horizon.

The assumption on $\phi$, that it is positive away from the boundary and satisfies (1.10), may seem restrictive if one hopes to apply our result to the Penrose inequality as described in [6]. However this is not the case. Three proposals for a coupling of the generalized Jang equation with other equations were outlined in [6], and it is the coupling with Bray's conformal flow which produces a warping factor $\phi$ that satisfies our hypotheses. The one missing ingredient here, in this paper, is that $\phi$ is fixed, and does not depend on $f$. Nevertheless, Theorem 1.1 provides the important first step in the difficult problem of analyzing the coupled Jang/conformal flow system. More precisely, given $\phi_{0}$ satisfying (1.10), Theorem 1.1 produces $f_{0}$, from which we may construct the Jang metric $\bar{g}_{0}=g+\phi_{0}^{2} d f_{0}^{2}$. According to [6], Bray's conformal flow with respect to $\bar{g}_{0}$ produces a new warping factor $\phi_{1}$, which will also satisfy the hypotheses of Theorem 1.1. We may then continue this procedure indefinitely to produce sequences of functions $\left\{\phi_{i}\right\},\left\{f_{i}\right\}$. The second and final step should entail 
establishing appropriate a priori estimates, to show that a subsequence converges to yield a solution of the coupled system of equations. This last step will be quite involved.

This paper is organized as follows. In Section 2 we derive local a priori estimates, and in Section 3 an important Harnack inequality is proved. These two results are then combined with further global estimates to establish the existence of solutions on complete initial data, in Section 4. In Section 5 we produce the blow-up solutions of Theorem 1.1, and construct appropriate super solutions. Lastly, the more difficult sub solutions are constructed in Section 6 and Theorem 1.1 is proven.

\section{A Priori Estimates for the Generalized Jang Equation}

In this section we begin the proof of existence for the generalized Jang equation. Our first goal is to prove the same existence and regularity result as obtained by Schoen and Yau [19], for arbitrary positive warping factor $\phi$. This is fairly straight forward, in that the methods of Schoen and Yau still apply with little modification. The primary difference is that (especially for applications) it is important to track precisely how the estimates depend on $\phi$ and its derivatives. The estimates of this section will also be helpful for the case in which $\phi$ is allowed to vanish, but for now we assume that $\phi$ is strictly positive.

Let us set the notation. Consider a hypersurface $\Sigma \subset\left(M \times \mathbb{R}, g+\phi^{2} d t^{2}\right)$ given by the graph of a function $t=f(x)$. This surface has induced metric $\bar{g}=g+\phi^{2} d f^{2}$, and inverse matrix

$$
\bar{g}^{i j}=g^{i j}-\frac{\phi^{2} f^{i} f^{j}}{1+\phi^{2}|\nabla f|^{2}}
$$

where $f^{i}=g^{i j} f_{j}$ and $f_{i}=\partial_{x^{i}} f$, with $x^{i}, i=1,2,3$, local coordinates on $M$. Throughout the paper, a bar will be placed over geometric quantities associated with $\Sigma$. For instance $\bar{\nabla}$ will denote the induced connection on $\Sigma$, whereas $\nabla$ will denote the connection on the ambient manifold $\left(M \times \mathbb{R}, g+\phi^{2} d t^{2}\right)$. The unit normal to $\Sigma$ is given by

$$
N=\frac{\nabla f-\phi^{-2} \partial_{t}}{\sqrt{\phi^{-2}+|\nabla f|^{2}}}
$$

and $X_{i}=\partial_{x^{i}}+f_{i} \partial_{t}, i=1,2,3$ form a basis for the tangent space. Moreover a calculation ([5], 6]) shows that the second fundamental form of $\Sigma$ is given by

$$
A_{i j}=\left\langle\nabla_{X_{i}} N, X_{j}\right\rangle=\frac{\phi \nabla_{i j} f+\phi_{i} f_{j}+\phi_{j} f_{i}+\phi^{2} f^{m} \phi_{m} f_{i} f_{j}}{\sqrt{1+\phi^{2}|\nabla f|^{2}}} .
$$

In this section it will be assumed that $\Sigma$ satisfies an equation of the form

$$
H-\operatorname{Tr} K=F,
$$

for some function $F$ to be specified, where $H=\bar{g}^{i j} A_{i j}$ is mean curvature and $\operatorname{Tr} K$ denotes the trace of $K$ over $\Sigma$. In what follows, as in (2.2), we will drop the subscript $\Sigma$ when denoting the mean curvature and trace operations with respect to $\Sigma$.

We first estimate the mean curvature and its derivatives.

Lemma 2.1. Suppose that the surface $\Sigma$ satisfies (2.2). Then

$$
|H| \leq|F|+c(1+|\nabla \log \phi|),
$$

and

$$
|\bar{\nabla} H| \leq c\left(1+|\nabla F|+|\nabla \log \phi|^{2}+\phi^{-1}\left|\nabla^{2} \phi\right|+|A|+|A||\nabla \log \phi|\right),
$$

where $c$ is a universal constant. 
Proof. First, by (2.2), we have

$$
H=\bar{g}^{i j}\left(k_{i j}+\frac{\phi^{2}\left(\phi^{l} f_{l}\right) f_{i} f_{j}}{\sqrt{1+\phi^{2}|\nabla f|^{2}}}\right)+F=\bar{g}^{i j} k_{i j}+\frac{\phi^{2}\left(\phi^{l} f_{l}\right)|\nabla f|^{2}}{\left(1+\phi^{2}|\nabla f|^{2}\right)^{3 / 2}}+F .
$$

This implies (2.3) easily.

Next, a simple differentiation yields

$$
\begin{aligned}
\partial_{a} H= & \bar{g}^{i j} k_{i j ; a}+\partial_{a} F+\left(\frac{-2 \phi \phi_{a} f^{i} f^{j}-2 \phi^{2} f^{i} \nabla_{a} f^{j}}{1+\phi^{2}|\nabla f|^{2}}\right) k_{i j} \\
& +\frac{\phi^{2} f^{i} f^{j}}{\left(1+\phi^{2}|\nabla f|^{2}\right)^{2}}\left(2 \phi \phi_{a}|\nabla f|^{2}+2 \phi^{2} f^{n} \nabla_{n a} f\right) k_{i j}+\frac{2 \phi^{2}\left(\phi^{m} f_{m}\right) f^{n} \nabla_{n a} f}{\left(1+\phi^{2}|\nabla f|^{2}\right)^{3 / 2}} \\
& +\frac{2 \phi \phi_{a}\left(\phi^{n} f_{n}\right)|\nabla f|^{2}+\phi^{2}\left(\nabla_{a} \phi^{n}\right) f_{n}|\nabla f|^{2}+\phi^{2} \phi^{n} \nabla_{n a} f|\nabla f|^{2}}{\left(1+\phi^{2}|\nabla f|^{2}\right)^{3 / 2}} \\
& -\frac{3}{2} \frac{\phi^{2}\left(\phi^{m} f_{m}\right)|\nabla f|^{2}}{\left(1+\phi^{2}|\nabla f|^{2}\right)^{5 / 2}}\left(2 \phi \phi_{a}|\nabla f|^{2}+2 \phi^{2} f^{n} \nabla_{n a} f\right) .
\end{aligned}
$$

However, by (2.1)

$$
\nabla_{i j} f=\phi^{-1}\left(\sqrt{1+\phi^{2}|\nabla f|^{2}} A_{i j}-\phi_{i} f_{j}-\phi_{j} f_{i}-\phi^{2}\left(\phi^{m} f_{m}\right) f_{i} f_{j}\right) .
$$

Thus, after substitution, some cancelations occur and we obtain

$$
\begin{aligned}
\partial_{a} H= & \bar{g}^{i j} k_{i j ; a}+\partial_{a} F+\left(\frac{2 \phi \phi_{i} f_{j} f_{a}}{1+\phi^{2}|\nabla f|^{2}}-\frac{2 \phi f_{i} A_{j a}}{\sqrt{1+\phi^{2}|\nabla f|^{2}}}+\frac{2 \phi^{3} f_{i} f_{j} f^{n} A_{a n}}{\left(1+\phi^{2}|\nabla f|^{2}\right)^{3 / 2}}\right) k^{i j} \\
& +\frac{\phi^{2}|\nabla f|^{2} f^{n} \nabla_{a n} \phi}{\left(1+\phi^{2}|\nabla f|^{2}\right)^{3 / 2}}-\frac{2 \phi\left(\phi^{m} f_{m}\right)^{2} f_{a}}{\left(1+\phi^{2}|\nabla f|^{2}\right)^{3 / 2}}-\frac{\phi|\nabla f|^{2}|\nabla \phi|^{2} f_{a}}{\left(1+\phi^{2}|\nabla f|^{2}\right)^{3 / 2}}-\frac{\phi|\nabla f|^{2}\left(\phi^{m} f_{m}\right) \phi_{a}}{\left(1+\phi^{2}|\nabla f|^{2}\right)^{3 / 2}} \\
& +A_{a n}\left(\frac{2 \phi\left(\phi^{m} f_{m}\right) f^{n}}{1+\phi^{2}|\nabla f|^{2}}+\frac{\phi|\nabla f|^{2} \phi^{n}}{1+\phi^{2}|\nabla f|^{2}}-\frac{3 \phi^{3}|\nabla f|^{2}\left(\phi^{m} f_{m}\right) f^{n}}{\left(1+\phi^{2}|\nabla f|^{2}\right)^{2}}\right) .
\end{aligned}
$$

This implies (2.4).

We point out that (2.3) yields a uniform pointwise estimate of the mean curvature. However, (2.4) illustrates that first derivatives of the mean curvature are estimated in terms of the second fundamental form, which is not yet controlled. In fact, estimating the second fundamental form will take up a major part of this section.

We next establish a $C^{0}$ estimate for the second fundamental form of $\Sigma$. This result will arise from a Moser iteration applied to the Simons identity. See also the paper [2], in which a similar result is proven in more general ambient geometries.

Theorem 2.2. Suppose that the surface $\Sigma$ satisfies (2.2). Then

$$
\sup _{\Sigma}|A| \leq c
$$

where $c$ depends on $|\log \phi|,|\nabla \log \phi|,\left|\nabla^{2} \log \phi\right|,|F|$, and $|N(F)|$.

Proof. The proof is lengthy and is divided into several steps.

Step 1. Derivation of Simons identity and several related inequalities. Recall the Ricci commutation formula and Codazzi equations:

$$
\bar{\nabla}_{a} \bar{\nabla}_{n} A_{i j}-\bar{\nabla}_{n} \bar{\nabla}_{a} A_{i j}=-A_{m j} \bar{R}_{i a n}^{m}-A_{i m} \bar{R}_{j a n}^{m},
$$


and

$$
\bar{\nabla}_{a} A_{i j}=\bar{\nabla}_{i} A_{a j}+R_{N j i a}
$$

Use these to obtain

$$
\begin{aligned}
\bar{\Delta} A_{i j} & =\bar{g}^{n a} \bar{\nabla}_{n} \bar{\nabla}_{a} A_{i j} \\
& =\bar{g}^{n a}\left(\bar{\nabla}_{n} \bar{\nabla}_{i} A_{a j}+\bar{\nabla}_{n} R_{N j i a}\right) \\
& =\bar{g}^{n a}\left(\bar{\nabla}_{i} \bar{\nabla}_{n} A_{a j}-A_{m j} \bar{R}_{a n i}^{m}-A_{a m} \bar{R}_{j n i}^{m}+\bar{\nabla}_{n} R_{N j i a}\right) \\
& =\bar{g}^{n a} \bar{\nabla}_{i}\left(\bar{\nabla}_{j} A_{n a}+R_{N a j n}\right)-\bar{g}^{n a}\left(A_{m j} \bar{R}_{a n i}^{m}+A_{a m} \bar{R}_{j n i}^{m}-\bar{\nabla}_{n} R_{N j i a}\right) \\
& =\bar{\nabla}_{i} \bar{\nabla}_{j} H+\bar{g}^{n a}\left(\bar{\nabla}_{i} R_{N a j n}+\bar{\nabla}_{n} R_{N j i a}-A_{m j} \bar{R}_{a n i}^{m}-A_{a m} \bar{R}_{j n i}^{m}\right) .
\end{aligned}
$$

Now use the Gauss equations

$$
\bar{R}_{i j n d}=R_{i j n d}+A_{i n} A_{j d}-A_{i d} A_{j n}
$$

to find

$$
\begin{aligned}
\bar{\Delta} A_{i j}= & \bar{\nabla}_{i} \bar{\nabla}_{j} H+\bar{g}^{n a}\left(\bar{\nabla}_{i} R_{N a j n}+\bar{\nabla}_{n} R_{N j i a}\right)-\bar{g}^{n a} \bar{g}^{m d}\left(A_{m j} R_{d a n i}+A_{a m} R_{d j n i}\right) \\
& -\bar{g}^{n a} \bar{g}^{m d} A_{m j}\left(A_{d n} A_{a i}-A_{d i} A_{a n}\right)-\bar{g}^{n a} \bar{g}^{m d} A_{a m}\left(A_{d n} A_{j i}-A_{i d} A_{j n}\right) .
\end{aligned}
$$

Therefore

$$
\bar{\Delta} A_{i j}=\bar{\nabla}_{i} \bar{\nabla}_{j} H-|A|^{2} A_{i j}+H A_{i m} A_{j}^{m}+G_{i j}
$$

where

$$
G_{i j}=\bar{g}^{n a}\left(\bar{\nabla}_{i} R_{N a j n}+\bar{\nabla}_{n} R_{N j i a}-\bar{g}^{m d} A_{m j} R_{d a n i}-\bar{g}^{m d} A_{a m} R_{d j n i}\right) .
$$

We then obtain

$$
\begin{aligned}
\frac{1}{2} \bar{\Delta}|A|^{2}= & \bar{g}^{i d} \bar{g}^{j a}\left(A_{d a} \bar{\Delta} A_{i j}+\bar{g}^{m n} \bar{\nabla}_{m} A_{d a} \bar{\nabla}_{n} A_{i j}\right) \\
= & \bar{g}^{i d} \bar{g}^{j a}\left(\bar{g}^{m n} \bar{\nabla}_{m} A_{d a} \bar{\nabla}_{n} A_{i j}+A_{d a} G_{i j}\right)-|A|^{4} \\
& +\bar{g}^{i d} \bar{g}^{j a}\left(H A_{d a} A_{i m} A_{j}^{m}+A_{d a} \bar{\nabla}_{i} \bar{\nabla}_{j} H\right) .
\end{aligned}
$$

This is the Simons identity for $\Sigma$.

Note that we also have

$$
\frac{1}{2} \bar{\Delta}|A|^{2}=|A| \bar{\Delta}|A|+|\bar{\nabla}| A||^{2}
$$

so that

$$
|A| \bar{\Delta}|A|=|\bar{\nabla} A|^{2}-|\bar{\nabla}| A||^{2}-|A|^{4}+A^{i j}\left(\bar{\nabla}_{i} \bar{\nabla}_{j} H+G_{i j}+H A_{i m} A_{j}^{m}\right) .
$$

Set

$$
T:=|\bar{\nabla} A|^{2}-\left.|\bar{\nabla}| A\right|^{2} .
$$

For the remainder of this paragraph we will work in an orthonormal basis. Then

$$
T=\sum_{i, j, n}\left(\bar{\nabla}_{n} A_{i j}\right)^{2}-|A|^{-2} \sum_{n}\left(\sum_{i, j} A_{i j} \bar{\nabla}_{n} A_{i j}\right)^{2},
$$

so that

$$
|A|^{2} T=\frac{1}{2} \sum_{i, j, n, d, m}\left(A_{i j} \bar{\nabla}_{n} A_{d m}-A_{d m} \bar{\nabla}_{n} A_{i j}\right)^{2} .
$$


By setting $n=i$ and $m=j$ and using the Schwarz inequality, we have

$$
\begin{aligned}
|A|^{2} T & =\frac{1}{2} \sum_{i, j, d}\left(A_{i j} \bar{\nabla}_{i} A_{d j}-A_{d j} \bar{\nabla}_{i} A_{i j}\right)^{2}+\frac{1}{2} \sum_{\substack{i, j, n, d, m \\
(n, m) \neq(i, j)}}\left(A_{i j} \bar{\nabla}_{n} A_{d m}-A_{d m} \bar{\nabla}_{n} A_{i j}\right)^{2} \\
& \geq \frac{1}{18} \sum_{d}\left(\sum_{i, j} A_{i j} \bar{\nabla}_{i} A_{d j}-\sum_{i, j} A_{d j} \bar{\nabla}_{i} A_{i j}\right)^{2}+\frac{1}{2} \sum_{\substack{i, j, n, d, m \\
(n, m) \neq(i, j)}}\left(A_{i j} \bar{\nabla}_{n} A_{d m}-A_{d m} \bar{\nabla}_{n} A_{i j}\right)^{2} .
\end{aligned}
$$

Moreover, according to the Codazzi equations (2.8),

$$
\sum_{i, j} A_{i j} \bar{\nabla}_{i} A_{d j}=\sum_{i, j} A_{i j} \bar{\nabla}_{d} A_{i j}+\sum_{i, j} A_{i j} R_{N j d i}
$$

and

It follows that

$$
\sum_{i, j} A_{d j} \bar{\nabla}_{i} A_{i j}=\sum_{j} A_{d j} \bar{\nabla}_{j} H+\sum_{i, j} A_{d j} R_{N i j i}
$$

$$
\begin{aligned}
|A|^{2} T \geq & \frac{1}{36} \sum_{l}\left(\sum_{i, j} A_{i j} \bar{\nabla}_{d} A_{i j}\right)^{2} \\
& -\frac{1}{18} \sum_{d}\left(\sum_{i, j} A_{i j} R_{N j d i}-\sum_{j} A_{d j} \bar{\nabla}_{j} H-\sum_{i, j} A_{d j} R_{N i j i}\right)^{2} \\
& +\frac{1}{2} \sum_{\substack{i, j, n, d, m \\
n, m) \neq(i, j)}}\left(A_{i j} \bar{\nabla}_{n} A_{d m}-A_{d m} \bar{\nabla}_{n} A_{i j}\right)^{2},
\end{aligned}
$$

after using $(a+b)^{2} \geq \frac{1}{2} a^{2}-b^{2}$. The definition of $T$ in (2.11) now yields

$$
\begin{aligned}
T \geq & \frac{1}{37} \sum_{i, j, n}\left(\bar{\nabla}_{n} A_{i j}\right)^{2} \\
& -\frac{36}{37} \cdot \frac{1}{18}|A|^{-2} \sum_{d}\left(\sum_{i, j} A_{i j} R_{N j d i}-\sum_{j} A_{d j} \bar{\nabla}_{j} H-\sum_{i, j} A_{d j} R_{N i j i}\right)^{2} \\
& +\frac{36}{37} \cdot \frac{1}{2}|A|^{-2} \sum_{\substack{i, j, n, d, m \\
n, m) \neq(i, j)}}\left(A_{i j} \bar{\nabla}_{n} A_{d m}-A_{d m} \bar{\nabla}_{n} A_{i j}\right)^{2} .
\end{aligned}
$$

Hence

$$
\begin{aligned}
|A| \bar{\Delta}|A| \geq & \frac{1}{37} \sum_{i, j, n}\left(\bar{\nabla}_{n} A_{i j}\right)^{2}-|A|^{4}+\sum_{i, j} A_{i j}\left(\bar{\nabla}_{i j} H+G_{i j}+\sum_{m} H A_{i m} A_{j m}\right) \\
& -\frac{2}{37}|A|^{-2} \sum_{d}\left(\sum_{i, j} A_{i j} R_{N j d i}-\sum_{j} A_{d j} \bar{\nabla}_{j} H-\sum_{i, j} A_{d j} R_{N i j i}\right)^{2} \\
& +\frac{2}{37}|A|^{-2} \sum_{\substack{i, j, n, d, m \\
(n, m) \neq(i, j)}}\left(A_{i j} \bar{\nabla}_{n} A_{d m}-A_{d m} \bar{\nabla}_{n} A_{i j}\right)^{2} .
\end{aligned}
$$


We can also write

$$
|A| \bar{\Delta}|A| \geq \frac{1}{37} \sum_{i, j, n}\left(\bar{\nabla}_{n} A_{i j}\right)^{2}-|A|^{4}-|H||A|^{3}+\sum_{i, j} A_{i j} \bar{\nabla}_{i j} H+G_{1},
$$

where

$$
\begin{aligned}
G_{1}:= & \sum_{i, j} A_{i j} G_{i j}-\frac{2}{37}|A|^{-2} \sum_{d}\left(\sum_{i, j} A_{i j} R_{N j d i}-\sum_{j} A_{d j} \bar{\nabla}_{j} H-\sum_{i, j} A_{d j} R_{N i j i}\right)^{2} \\
& +\frac{2}{37}|A|^{-2} \sum_{\substack{i, j, n, d, m \\
n, m) \neq(i, j)}}\left(A_{i j} \bar{\nabla}_{n} A_{d m}-A_{d m} \bar{\nabla}_{n} A_{i j}\right)^{2} .
\end{aligned}
$$

Step 2. An $L^{4}$-estimate for the second fundamental form. Taking two traces of the Gauss equations (2.9) produces

$$
\bar{R}=\bar{g}^{i n} \bar{g}^{j m} R_{i j n m}+H^{2}-|A|^{2} .
$$

Moreover, equation (2.2) implies that

$$
\bar{R}+|A|^{2}=\bar{g}^{i n} \bar{g}^{j m} R_{i j n m}+(\operatorname{Tr} K+F)^{2} .
$$

Recall the formula for the scalar curvature of surface $\Sigma$ (see [5], 6])

$$
\begin{aligned}
\bar{R}= & 2(\mu-J(w))+|A-K|^{2}+2|q|^{2}-\frac{2}{\phi} \overline{\operatorname{div}}(\phi q) \\
& +H^{2}-(\operatorname{Tr} K)^{2}+2 K(N, N)(H-\operatorname{Tr} K)+2 N(H-\operatorname{Tr} K) .
\end{aligned}
$$

By combining the previous two formulas we then have

$$
\begin{aligned}
& |A|^{2}+|A-K|^{2}+2(\mu-J(w))+2|q|^{2} \\
= & \frac{2}{\phi} \overline{\operatorname{div}}(\phi q)-(2 \operatorname{Tr} K+F) F-2 K(N, N) F \\
& -2 N(F)+\bar{g}^{i n} \bar{g}^{j m} R_{i j n m}+(\operatorname{Tr} K+F)^{2} .
\end{aligned}
$$

Note that

$$
|A-K|^{2}=|A|^{2}+|K|^{2}-2\langle A, K\rangle
$$

and set

$$
G_{2}:=2\langle A, K\rangle-|K|^{2}-2 K(N, N) F-2 N(F)+(\operatorname{Tr} K)^{2}-2(\mu-J(w))+\bar{g}^{i n} \bar{g}^{j m} R_{i j n m} .
$$

Then, (2.13) becomes

$$
2|A|^{2}=\frac{2}{\phi} \overline{\operatorname{div}}(\phi q)-2|q|^{2}+G_{2} .
$$

Let $\psi \in C_{c}^{\infty}(\Sigma)$. Multiply (2.14) by $\phi \psi^{2}$ and integrate by parts to find

$$
\begin{aligned}
\int_{\Sigma} 2 \phi \psi^{2}|A|^{2} & =\int_{\Sigma}-4 \phi \psi\langle\bar{\nabla} \psi, q\rangle-2 \phi \psi^{2}|q|^{2}+\phi \psi^{2} G_{2} \\
& \leq \int_{\Sigma} 2 \phi|\bar{\nabla} \psi|^{2}+\phi \psi^{2} G_{2} .
\end{aligned}
$$

Now replace $\psi$ with $|A|^{p} \psi$ to get

$$
\int_{\Sigma} \phi \psi^{2}|A|^{2+2 p} \leq \int_{\Sigma} \phi\left|\bar{\nabla}\left(|A|^{p} \psi\right)\right|^{2}+\frac{1}{2} \phi \psi^{2} G_{2}|A|^{2 p} .
$$


Expand and integrate the first term by parts:

$$
\begin{aligned}
\int_{\Sigma} \phi\left|\bar{\nabla}\left(|A|^{p} \psi\right)\right|^{2} & =\int_{\Sigma} \phi|A|^{2 p}|\bar{\nabla} \psi|^{2}+2 p \phi \psi|A|^{2 p-1}\langle\bar{\nabla}|A|, \bar{\nabla} \psi\rangle+\left.\left.\phi \psi^{2}|\bar{\nabla}| A\right|^{p}\right|^{2} \\
& =\int_{\Sigma} \phi|A|^{2 p}|\bar{\nabla} \psi|^{2}-\frac{1}{2} \phi \psi^{2} \bar{\Delta}|A|^{2 p}+\left.\left.\phi \psi^{2}|\bar{\nabla}| A\right|^{p}\right|^{2}-\psi^{2}\left\langle\bar{\nabla} \phi, \bar{\nabla}|A|^{2 p}\right\rangle \\
& =\int_{\Sigma} \phi|A|^{2 p}|\bar{\nabla} \psi|^{2}-\phi \psi^{2}|A|^{p} \bar{\Delta}|A|^{p}-2 p \psi^{2}|A|^{2 p-1}\langle\bar{\nabla} \phi, \bar{\nabla}|A|\rangle .
\end{aligned}
$$

Since

$$
|A|^{p} \bar{\Delta}|A|^{p}=p|A|^{2 p-1} \bar{\Delta}|A|+p(p-1)|A|^{2 p-2}|\bar{\nabla}| A||^{2},
$$

we then have

$$
\begin{aligned}
& \int_{\Sigma} \phi \psi^{2}\left(|A|^{2 p-1}\left(p \bar{\Delta}|A|+|A|^{3}\right)+\left.p(p-1)|A|^{2 p-2}|\bar{\nabla}| A\right|^{2}\right) \\
\leq & \int_{\Sigma} \phi|A|^{2 p}|\bar{\nabla} \psi|^{2}-2 p \psi^{2}|A|^{2 p-1}\langle\bar{\nabla} \phi, \bar{\nabla}|A|\rangle+\frac{1}{2} \phi \psi^{2}|A|^{2 p} G_{2} \\
= & \int_{\Sigma} \phi|A|^{2 p}|\bar{\nabla} \psi|^{2}+2 \psi|A|^{2 p}\langle\bar{\nabla} \psi, \bar{\nabla} \phi\rangle+\phi \psi^{2}|A|^{2 p}\left(\frac{1}{2} G_{2}+\phi^{-1} \bar{\Delta} \phi\right) \\
\leq & \int_{\Sigma} 2 \phi|A|^{2 p}|\bar{\nabla} \psi|^{2}+\phi \psi^{2}|A|^{2 p}\left(\frac{1}{2} G_{2}+\phi^{-2}|\bar{\nabla} \phi|^{2}+\phi^{-1} \bar{\Delta} \phi\right) .
\end{aligned}
$$

By using (2.12) for $|A|\left(\bar{\Delta}|A|+|A|^{3}\right)$ and setting $p=1$ in the above expression, it follows that

$$
\begin{aligned}
\int_{\Sigma} \frac{1}{37} \phi \psi^{2}|\bar{\nabla} A|^{2} \leq & \int_{\Sigma} 2 \phi|A|^{2}|\bar{\nabla} \psi|^{2}+\phi \psi^{2}|A|^{2}\left(\frac{1}{2} G_{2}+\phi^{-2}|\bar{\nabla} \phi|^{2}+\phi^{-1} \bar{\Delta} \phi\right) \\
& +\int_{\Sigma} \phi \psi^{2}\left(|H||A|^{3}-G_{1}-A^{i j} \bar{\nabla}_{i j} H\right) .
\end{aligned}
$$

Integrating by parts and applying the Schwarz inequality then yields

$$
\begin{aligned}
\int_{\Sigma} c^{-1} \phi \psi^{2}|\bar{\nabla} A|^{2} \leq & \int_{\Sigma} c \phi|A|^{2}|\bar{\nabla} \psi|^{2}+\phi \psi^{2}|A|^{2}\left(\frac{1}{2} G_{2}+c|\bar{\nabla} \log \phi|^{2}\right) \\
& +\int_{\Sigma} \phi \psi^{2}\left(|H||A|^{3}-G_{1}+c|\bar{\nabla} H|^{2}\right) .
\end{aligned}
$$

Above and in what follows, $c>0$ will always denote an appropriately large constant. Although there are derivatives of the Riemann tensor contained within the expression for $G_{1}$, these may be integrated by parts so that

$$
\begin{aligned}
\int_{\Sigma} c^{-1} \phi \psi^{2}|\bar{\nabla} A|^{2} \leq & \int_{\Sigma} \phi|A|^{2}|\bar{\nabla} \psi|^{2} \\
& +\int_{\Sigma} \phi \psi^{2}\left(\mid \text { Riem }\left.\right|^{2}+|\bar{\nabla} H|^{2}+|\nabla \log \phi|^{2} \mid \text { Riem }\left.\right|^{2}\right) \\
& +\int_{\Sigma} \phi \psi^{2}|A|^{2}\left(1+\mid \text { Riem }\left.|+| \nabla \log \phi\right|^{2}+|N(F)|+(1+|\nabla \log \phi|)|F|\right) \\
& +\int_{\Sigma} \phi \psi^{2}|A|^{3}(|H|+|\nabla \log \phi|) .
\end{aligned}
$$


Now observe that according to (2.15) with $p=1$,

$$
\begin{aligned}
\int_{\Sigma} \phi \psi^{2}|A|^{4} & \leq \int_{\Sigma} \phi|\bar{\nabla}(|A| \psi)|^{2}+\frac{1}{2} \phi \psi^{2} G_{2}|A|^{2} \\
& \leq \int_{\Sigma} 2 \phi|A|^{2}|\bar{\nabla} \psi|^{2}+\phi \psi^{2}\left(\left.2|\bar{\nabla}| A\right|^{2}+\frac{1}{2} G_{2}|A|^{2}\right) .
\end{aligned}
$$

Also

$$
\begin{aligned}
\left.|\bar{\nabla}| A\right|^{2} & =\bar{g}^{i j} \partial_{i}|A| \partial_{j}|A| \\
& =\bar{g}^{i j}\left(|A|^{-1} A^{a b} \bar{\nabla}_{i} A_{a b}\right)\left(|A|^{-1} A^{c d} \bar{\nabla}_{j} A_{c d}\right) \\
& \leq c|\bar{\nabla} A|^{2} .
\end{aligned}
$$

Therefore

$$
\int_{\Sigma} \phi \psi^{2}|A|^{4} \leq \int_{\Sigma} 2 \phi|A|^{2}|\bar{\nabla} \psi|^{2}+\frac{1}{2} \phi \psi^{2} G_{2}|A|^{2}+c \phi \psi^{2}|\bar{\nabla} A|^{2} .
$$

Combining this with (2.16) then yields

$$
\begin{aligned}
\int_{\Sigma} c^{-1} \phi \psi^{2}|A|^{4} \leq & \int_{\Sigma} \phi|A|^{2}|\bar{\nabla} \psi|^{2} \\
& +\int_{\Sigma} \phi \psi^{2}\left(\mid \text { Riem }\left.\right|^{2}+|\bar{\nabla} H|^{2}+|\nabla \log \phi|^{2} \mid \text { Riem }\left.\right|^{2}\right) \\
& +\int_{\Sigma} \phi \psi^{2}|A|^{2}\left(1+\mid \text { Riem }\left.|+| \nabla \log \phi\right|^{2}+|N(F)|+(1+|\nabla \log \phi|)|F|\right) \\
& +\int_{\Sigma} \phi \psi^{2}|A|^{3}(|H|+|\nabla \log \phi|) .
\end{aligned}
$$

Now replace $\psi$ with $\psi^{2}$ and apply the Schwarz inequality to obtain

$$
\int_{\Sigma} c^{-1} \phi \psi^{4}|A|^{4} \leq \int_{\Sigma} \phi|\bar{\nabla} \psi|^{4}+\phi \psi^{4}\left(1+|H|^{4}+|\bar{\nabla} H|^{2}+\mid \text { Riem }\left.\right|^{4}+|\nabla \log \phi|^{4}+|F|^{4}+|N(F)|^{2}\right) .
$$

Let $B_{r_{0}}^{4}\left(x_{0}\right)$ be a geodesic ball in $\left(M \times \mathbb{R}, g+\phi^{2} d t^{2}\right)$ centered at a point $x_{0} \in \Sigma$, with $r_{0}$ less than the injectivity radius. Let $r$ be the distance function in $M \times \mathbb{R}$ and choose the cut-off function $\psi$ to be a function of $r$ such that $\psi(r)=1$ for $r \leq r_{0} / 2$ and $\psi(r)=0$ for $r \geq r_{0}$. Since $|\nabla r|=1$ and hence $|\bar{\nabla} r| \leq 1$, we may further choose $\psi$ so that $|\psi| \leq 1$ and $|\bar{\nabla} \psi| \leq 3 r_{0}^{-1}$. In order to bound the volume of $\Sigma \cap B_{r_{0}}^{4}\left(x_{0}\right)$, note that by (2.3)

$$
\left|\operatorname{div}_{M \times \mathbb{R}}(N)\right|=|H| \leq|F|+c(1+|\nabla \log \phi|) .
$$

Upon an integration by parts over the region $B_{r_{0}}^{4}\left(x_{0}\right) \cap\{(x, t) \mid t<f(x)\}$, we obtain

$$
\operatorname{Vol}\left(\Sigma \cap B_{r_{0}}^{4}\left(x_{0}\right)\right) \leq c r_{0}^{3}
$$

where $c$ depends on $|F|$ and $|\nabla \log \phi|$. Applying this result together with (2.17) then yields

$$
\int_{\Sigma \cap B_{\frac{r_{0}}{2}}^{4}\left(x_{0}\right)}|A|^{4} \leq c,
$$

where $c$ depends on $|F|,|N(F)|,|H|,|\bar{\nabla} H|,|\nabla \log \phi|, \mid$ Riem $\mid, \frac{\max \phi}{\min \phi}$, and $r_{0}^{-1}$. This is the desired $L^{4}$-estimate for the second fundamental form.

Step 3. A pointwise estimate of the second fundamental form. Set $u=|A|^{2}+1$. Then (2.10) yields

$$
\bar{\Delta} u \geq-c\left(1+|A|^{2}+|F|^{2}+|\nabla \log \phi|^{2}\right) u+2|\bar{\nabla} A|^{2}+2 A^{i j}\left(\bar{\nabla}_{i j} H+G_{i j}\right) \text {. }
$$


Multiply both sides by a nonnegative function $\xi \in C_{c}^{\infty}\left(\Sigma \cap B_{\frac{r_{0}}{2}}^{4}\left(x_{0}\right)\right)$ and integrate by parts to find

$$
\begin{aligned}
0 \geq & \int_{\Sigma}\langle\bar{\nabla} \xi, \bar{\nabla} u\rangle-c\left(1+|A|^{2}+|F|^{2}+|\nabla \log \phi|^{2}\right) \xi u-2 A^{i j} \bar{\nabla}_{i} \xi \bar{\nabla}_{j} H \\
& +\int_{\Sigma} 2 \xi\left(|\bar{\nabla} A|^{2}+A^{i j} G_{i j}-\bar{\nabla}_{i} A^{i j} \bar{\nabla}_{j} H\right) .
\end{aligned}
$$

Although $G_{i j}$ contains first derivatives of the Riemann tensor, these derivatives may be integrated by parts. The first derivatives of $A$ which result from this process may be absorbed into $|\bar{\nabla} A|^{2}$ to yield a more simple expression

$$
0 \geq \int_{\Sigma} \bar{\nabla} \xi \cdot \bar{\nabla} u+D^{i}\left(\bar{\nabla}_{i} \xi\right) u+D \xi u
$$

where

$$
D^{i}=-2 u^{-1} A^{i j} \bar{\nabla}_{j} H-2 u^{-1} A^{i j} \bar{g}^{n m} R_{N n j m}-2 u^{-1} A^{n m} \bar{g}^{i j} R_{N n m j},
$$

and

$$
D=-c\left(1+|A|^{2}+|F|^{2}+|\nabla \log \phi|^{2}+u^{-1}|\bar{\nabla} H|^{2}+u^{-1}|A|^{2} \mid \text { Riem }\left|+u^{-1}\right| \text { Riem }\left.\right|^{2}\right) .
$$

By (2.4), we have

$$
\sup _{\Sigma \cap B_{\frac{r_{0}}{2}}^{4}\left(x_{0}\right)}\left|D^{i}\right|^{2}+\int_{\Sigma \cap B_{\frac{r_{0}}{2}}^{4}\left(x_{0}\right)} D^{2} \leq c .
$$

We now show that the Sobolev inequality holds on $\Sigma \cap B_{r_{0}}^{4}\left(x_{0}\right)$. The volume estimate (2.18) allows an application of the Hoffman and Spruck inequality [12] for a sufficiently small $r_{0}$. In particular, for any function $\psi \in C_{c}^{\infty}\left(\Sigma \cap B_{r_{0}}^{4}\left(x_{0}\right)\right)$, we have

$$
\left(\int_{\Sigma} \psi^{6}\right)^{1 / 3} \leq c \int_{\Sigma}\left(|\bar{\nabla} \psi|^{2}+H^{2} \psi^{2}\right) .
$$

Moreover, by (2.3) and Holder's inequality

$$
c^{-1}\left(\int_{\Sigma} \psi^{6}\right)^{1 / 3} \leq \int_{\Sigma}|\bar{\nabla} \psi|^{2}+r_{0}^{2}\left(1+|F|^{2}+|\nabla \log \phi|^{2}\right)\left(\int_{\Sigma} \psi^{6}\right)^{1 / 3} .
$$

Thus, if $r_{0}$ is sufficiently small we obtain the desired Sobolev inequality

$$
\left(\int_{\Sigma} \psi^{6}\right)^{1 / 3} \leq c \int_{\Sigma}|\bar{\nabla} \psi|^{2}
$$

A Moser iteration [11] can now be applied with the help of (2.20) and (2.21), since $2>\frac{1}{2} \operatorname{dim} \Sigma=\frac{3}{2}$. It follows that

$$
u\left(x_{0}\right) \leq c\left(\int_{\Sigma \cap B_{\frac{r_{0}}{2}}^{4}\left(x_{0}\right)} u^{2}\right)^{1 / 2} .
$$

Now the desired estimate (2.6) follows from the definition of $u$ and (2.19).

As a corollary of Theorem [2.2, we have a pointwise gradient estimate for the mean curvature, which follows easily from (2.4) and (2.6). 
Corollary 2.3. Suppose that the surface $\Sigma$ satisfies (2.2). Then

$$
\sup _{\Sigma}|\bar{\nabla} H| \leq c
$$

where $c$ depends on $|\log \phi|,|\nabla \log \phi|,\left|\nabla^{2} \log \phi\right|,|F|$, and $|N(F)|$.

Let $y^{1}, y^{2}, y^{3}, y^{4}$ be normal coordinates for the warped product metric near $x_{0} \in \Sigma$, that is

$$
\widehat{g}=g+\phi^{2} d t^{2}=\widehat{g}_{a b} d y^{a} d y^{b}, \quad \widehat{g}_{a b}(0)=\delta_{a b}, \quad \partial_{y^{c}} \widehat{g}_{a b}(0)=0 .
$$

Suppose that $\partial_{y^{i}} \in T_{x_{0}} \Sigma, i=1,2,3$ so that near $x_{0}, \Sigma$ is given by a graph $y^{4}=w(y), y=\left(y^{1}, y^{2}, y^{3}\right)$. Equation (2.2) can now be written as

$$
\sum_{a, b=1}^{4}\left(\widehat{g}^{a b}-\frac{W^{a} W^{b}}{|\nabla W|^{2}}\right)\left(\frac{\nabla_{a} \nabla_{b} W}{|\nabla W|}-K_{a b}\right)=F,
$$

where $W(Y)=w(y)-y^{4}, Y=\left(y^{1}, y^{2}, y^{3}, y^{4}\right)$. To see this first observe that $Y_{i}=\partial_{y^{i}}+w_{, i} \partial_{y^{4}}$, $i=1,2,3$, form a basis for the tangent space to $\Sigma$. Let $N=N_{a} d y^{a}$ be the unit normal written as a 1-form. Then

$$
0=\widehat{g}\left(Y_{i}, N\right)=Y_{i}^{a} N_{a}=\sum_{a=1}^{3} \delta_{i}^{a} N_{a}+w_{, i} N_{4}=N_{i}+w_{, i} N_{4}
$$

It follows that

$$
N=\frac{\sum_{a=1}^{3} w_{, a} d y^{a}-d y^{4}}{|\nabla W|}:=\frac{\widetilde{N}}{|\widetilde{N}|} .
$$

The second fundamental form, in these coordinates, is then given by

$$
A_{i j}=\widehat{g}\left(\nabla_{Y_{i}} N, Y_{j}\right)=\frac{\nabla_{i} \tilde{N}_{j}}{|\widetilde{N}|}=\frac{\nabla_{i} \nabla_{j} W}{|\nabla W|} .
$$

Moreover, the induced metric on $\Sigma$ is $\widehat{g}_{a b}-N_{a} N_{b}$, with inverse

$$
\widehat{g}^{a b}-N^{a} N^{b}=\widehat{g}^{a b}-\frac{W^{a} W^{b}}{|\nabla W|^{2}} .
$$

This yields the desired expression for the generalized Jang equation.

Parametric estimates may now be obtained as another application of Theorem 2.2 .

Theorem 2.4. Suppose that the surface $\Sigma$ satisfies (2.2). Then there exist constants $\rho>0$ and $c$ independent of $\Sigma$, but dependent on $|\log \phi|_{C^{2, \alpha}}$ and $|F|_{C^{1, \alpha}}$, such that $\Sigma \cap B_{\rho}^{4}\left(x_{0}\right) \subset\left\{Y \mid y^{4}=w(y)\right\}$ and $|w|_{C^{3, \alpha}\left(B_{\rho}\right)} \leq c$.

Proof. Observe that (2.23) is a strictly elliptic equation for $w$ having the following structure

$$
\sum_{i, j=1}^{3} B^{i j}(y, w, \partial w) w_{y^{i} y^{j}}=C(y, w, \partial w)
$$

where $B^{i j}(0,0,0)=\delta^{i j}$. Since the second fundamental form is uniformly bounded and

$$
|A|^{2}=\sum_{a, b, c, d=1}^{4}\left(\widehat{g}^{a c}-\frac{W^{a} W^{c}}{|\nabla W|^{2}}\right)\left(\widehat{g}^{b d}-\frac{W^{b} W^{d}}{|\nabla W|^{2}}\right)\left(\frac{\nabla_{a} \nabla_{b} W}{|\nabla W|}\right)\left(\frac{\nabla_{c} \nabla_{d} W}{|\nabla W|}\right),
$$


we obtain

$$
\sum_{i, j=1}^{3}\left(w_{y^{i} y^{j}}\right)^{2} \leq c\left(1+\sum_{i=1}^{3}\left(w_{y^{i}}\right)^{2}\right)^{3},
$$

near $y=0$. Now by some simple calculus,

$$
\sup _{|y| \leq \rho}\left(|w(y)|+|\partial w(y)|+\left|\partial^{2} w(y)\right|\right) \leq c .
$$

Therefore the $C^{3, \alpha}$ estimates follow from Schauder's theory and the $C^{2}$ estimates above.

Lastly, we note that $\Sigma$ may be expressed as a graph for sufficiently small (but uniform) $\rho>0$. This follows directly from the pointwise bound on the second fundamental form established in Theorem 2.2 ,

\section{The Harnack Inequality}

In this section it will be shown that the quantity $\left\langle\partial_{t}, N\right\rangle$ satisfies a homogeneous elliptic equation with bounded coefficients, when $|A|$ is pointwise bounded. From this we immediately obtain the Harnack inequality as in [19], which is used extensively. The proof here consists of a long calculation. Although the resulting equation has the same structure as that of [19], we cannot simply cite this reference, as the calculations carried out here must be done in the warped product setting.

We begin by recalling that the vector fields $X_{i}=\partial_{i}+f_{i} \partial_{t}$ form a basis for the tangent space to the surface $\Sigma=\{t=f(x)\}$, and that the vector field

$$
N=\frac{f^{m} \partial_{m}-\phi^{-2} \partial_{t}}{\sqrt{\phi^{-2}+|\nabla f|^{2}}}
$$

is its unit normal. Then

$$
\left\langle\partial_{t}, N\right\rangle=\frac{-1}{\sqrt{\phi^{-2}+|\nabla f|^{2}}}
$$

Our goal will be to calculate the Laplacian

$$
\bar{\Delta}\left\langle\partial_{t}, N\right\rangle=\bar{g}^{p j} \bar{\nabla}_{X_{p}} \bar{\nabla}_{X_{j}}\left\langle\partial_{t}, N\right\rangle
$$

in terms of $\left\langle\partial_{t}, N\right\rangle$. As usual, in this notation, an over line bar indicates that the particular geometric quantity is with respect to the induced metric on $\Sigma$.

The following lemma may be obtained from the Jacobi equation, as indicated in [3]. However here, we carry out a different (and longer) proof, as some of the calculations can be used later.

Lemma 3.1. For any $\Sigma \subset\left(M \times \mathbb{R}, g+\phi^{2} d t^{2}\right)$, not necessarily satisfying (2.2),

$$
\bar{\Delta}\left\langle\partial_{t}, N\right\rangle+\left(|A|^{2}+N(H)+R_{N N}\right)\left\langle\partial_{t}, N\right\rangle=0 .
$$

Proof. First observe that

$$
A_{i j}=\left\langle X_{i}, \nabla_{X_{j}} N\right\rangle
$$

so that

$$
\nabla_{X_{j}} N=A_{j p} \bar{g}^{p a} X_{a}
$$

It follows that

$$
\bar{\nabla}_{X_{j}}\left\langle\partial_{t}, N\right\rangle=\left\langle\nabla_{X_{j}} \partial_{t}, N\right\rangle+\left\langle\partial_{t}, X_{m}\right\rangle \bar{g}^{m n} A_{j n}
$$

and

$$
\bar{\nabla}_{X_{p}} \bar{\nabla}_{X_{j}}\left\langle\partial_{t}, N\right\rangle=\bar{\nabla}_{X_{p}}\left\langle\nabla_{X_{j}} \partial_{t}, N\right\rangle+\bar{g}^{m n}\left(\bar{\nabla}_{p} A_{j n}\right)\left\langle\partial_{t}, X_{m}\right\rangle+\bar{g}^{m n} A_{j n} \bar{\nabla}_{X_{p}}\left\langle\partial_{t}, X_{m}\right\rangle .
$$


The Codazzi equations (2.8) yield

$$
\bar{\nabla}_{p} A_{j n}=\bar{\nabla}_{n} A_{j p}+R_{N X_{j} X_{n} X_{p}} .
$$

Moreover

$$
\begin{aligned}
\bar{\nabla}_{X_{p}}\left\langle\partial_{t}, X_{m}\right\rangle & =X_{p}\left\langle\partial_{t}, X_{m}\right\rangle-\bar{\Gamma}_{p m}^{a}\left\langle\partial_{t}, X_{a}\right\rangle \\
& =\left\langle\nabla_{X_{p}} \partial_{t}, X_{m}\right\rangle+\left\langle\partial_{t}, \nabla_{X_{p}} X_{m}\right\rangle-\bar{\Gamma}_{p m}^{a}\left\langle\partial_{t}, X_{a}\right\rangle
\end{aligned}
$$

and

Thus

$$
\nabla_{X_{p}} X_{m}=\bar{\Gamma}_{p m}^{a} X_{a}-A_{p m} N
$$

$$
\begin{aligned}
\bar{\Delta}\left\langle\partial_{t}, N\right\rangle= & \bar{g}^{p j} \bar{\nabla}_{X_{p}} \bar{\nabla}_{X_{j}}\left\langle\partial_{t}, N\right\rangle \\
= & \bar{g}^{p j} \bar{\nabla}_{X_{p}}\left\langle\nabla_{X_{j}} \partial_{t}, N\right\rangle-|A|^{2}\left\langle\partial_{t}, N\right\rangle \\
& +\bar{g}^{m n}\left(\bar{\nabla}_{n} H+R_{N X_{n}}\right)\left\langle\partial_{t}, X_{m}\right\rangle+A^{p m}\left\langle\nabla_{X_{p}} \partial_{t}, X_{m}\right\rangle .
\end{aligned}
$$

The right-hand side will be calculated term by term.

We first claim that

$$
\bar{g}^{m n}\left(\bar{\nabla}_{n} H+R_{N X_{n}}\right)\left\langle\partial_{t}, X_{m}\right\rangle=-\left\langle\partial_{t}, N\right\rangle\left(\phi^{-1} \Delta \phi+N(H)+R_{N N}\right) .
$$

To prove this, write

$$
\partial_{t}=\left\langle\partial_{t}, N\right\rangle N+\bar{g}^{m n}\left\langle\partial_{t}, X_{m}\right\rangle X_{n}
$$

and observe that

$$
\bar{g}^{m n}\left(\bar{\nabla}_{n} H+R_{N X_{n}}\right)\left\langle\partial_{t}, X_{m}\right\rangle=\operatorname{Ric}\left(N, \partial_{t}\right)+\partial_{t} H-\left\langle\partial_{t}, N\right\rangle\left(R_{N N}+N(H)\right) .
$$

The desired result follows since $\partial_{t} H=0$ and

$$
\operatorname{Ric}\left(N, \partial_{t}\right)=-\left\langle\partial_{t}, N\right\rangle \phi^{-1} \Delta \phi .
$$

To see this last assertion, use the fact that

$$
\Gamma_{44}^{4}=\Gamma_{i j}^{4}=\Gamma_{i 4}^{j}=0, \quad \Gamma_{i 4}^{4}=(\log \phi)_{i}, \quad \Gamma_{44}^{i}=-\phi \phi^{i},
$$

to calculate

$$
R_{4 i j k}=0, \quad R_{4 i 4 j}=-\phi \nabla_{i j} \phi .
$$

Here 4 indicates the $t$-coordinate. This implies that

$$
R_{4 i}=0, \quad R_{44}=-\phi \Delta \phi,
$$

and hence

$$
\operatorname{Ric}\left(N, \partial_{t}\right)=-\left\langle\partial_{t}, N\right\rangle \operatorname{Ric}\left(f^{m} \partial_{m}-\phi^{-2} \partial_{t}, \partial_{t}\right)=-\left\langle\partial_{t}, N\right\rangle \phi^{-1} \Delta \phi .
$$

This finishes the proof of (3.3).

Next, with the help of (3.4), a straightforward calculation yields

$$
\nabla_{X_{p}} \partial_{t}=(\log \phi)_{p} \partial_{t}-\phi f_{p} \phi^{m} \partial_{m}
$$

Therefore

$$
\left\langle\nabla_{X_{p}} \partial_{t}, X_{m}\right\rangle=\phi\left(f_{m} \phi_{p}-f_{p} \phi_{m}\right) .
$$

Since this is an antisymmetric tensor, and $A^{p m}$ is symmetric, we obtain

$$
A^{p m}\left\langle\nabla_{X_{p}} \partial_{t}, X_{m}\right\rangle=0
$$

Alternatively, since $\partial_{t}$ is a Killing field, it follows immediately that $\left\langle\nabla_{X_{p}} \partial_{t}, X_{m}\right\rangle$ is antisymmetric. 
Lastly, we claim that

$$
\bar{g}^{p j} \bar{\nabla}_{X_{p}}\left\langle\nabla_{X_{j}} \partial_{t}, N\right\rangle=\left\langle\partial_{t}, N\right\rangle \phi^{-1} \Delta \phi .
$$

First observe that since $\partial_{t}$ is a Killing field,

$$
\left\langle\nabla_{X_{j}} \partial_{t}, N\right\rangle=-\left\langle\nabla_{N} \partial_{t}, X_{j}\right\rangle .
$$

Furthermore

$$
\nabla_{N} \partial_{t}=\nabla_{\partial_{t}} N-L_{\partial_{t}} N=\nabla_{\partial_{t}} N
$$

where $L$ denotes Lie differentiation. It follows that

$$
\left\langle\nabla_{X_{j}} \partial_{t}, N\right\rangle=-\left\langle\nabla_{\partial_{t}} N, X_{j}\right\rangle .
$$

Now calculate

$$
\begin{aligned}
\nabla_{\partial_{t}} N & =\left(\phi^{-2}+|\nabla f|^{2}\right)^{-1 / 2}\left(f^{i} \Gamma_{i 4}^{a} \partial_{a}-\phi^{-2} \Gamma_{44}^{a} \partial_{a}\right) \\
& =-\left\langle\partial_{t}, N\right\rangle\left(f^{i}(\log \phi)_{i} \partial_{t}+\phi^{-1} \phi^{i} \partial_{i}\right) \\
& =-\left\langle\partial_{t}, N\right\rangle(\log \phi)^{i} X_{i},
\end{aligned}
$$

to find

We then have

$$
\bar{g}^{p j}\left\langle\nabla_{\partial_{t}} N, X_{j}\right\rangle=-\left\langle\partial_{t}, N\right\rangle(\log \phi)^{p}
$$

$$
\begin{aligned}
\bar{\nabla}_{X_{p}}\left(\bar{g}^{p j}\left\langle\nabla_{\partial_{t}} N, X_{j}\right\rangle\right) & =X_{p}\left(\bar{g}^{p j}\left\langle\nabla_{\partial_{t}} N, X_{j}\right\rangle\right)+\bar{\Gamma}_{p n}^{p} \bar{g}^{n j}\left\langle\nabla_{\partial_{t}} N, X_{j}\right\rangle \\
& =-\left(\partial_{p}\left\langle\partial_{t}, N\right\rangle\right)(\log \phi)^{p}-\left\langle\partial_{t}, N\right\rangle \partial_{p}(\log \phi)^{p}-\left\langle\partial_{t}, N\right\rangle \bar{\Gamma}_{p n}^{p}(\log \phi)^{n} \\
& =-\left\langle\partial_{t}, N\right\rangle \Delta(\log \phi)+\left\langle\partial_{t}, N\right\rangle\left(\Gamma_{p n}^{p}-\bar{\Gamma}_{p n}^{p}\right)(\log \phi)^{n}-\left(\partial_{p}\left\langle\partial_{t}, N\right\rangle\right)(\log \phi)^{p} .
\end{aligned}
$$

According to a calculation in [5] (page 760),

Moreover, by (2.1),

$$
\Gamma_{p j}^{n}-\bar{\Gamma}_{p j}^{n}=\phi \phi^{n} f_{p} f_{j}-\frac{f^{n} A_{p j}}{\sqrt{\phi^{-2}+|\nabla f|^{2}}} .
$$

$$
\begin{aligned}
-\partial_{p}\left\langle\partial_{t}, N\right\rangle & =\partial_{p}\left(\phi^{-2}+|\nabla f|^{2}\right)^{-1 / 2} \\
& =\frac{\phi^{-3} \phi_{p}-f^{n} \nabla_{p n} f}{\left(\phi^{-2}+|\nabla f|^{2}\right)^{3 / 2}} \\
& =\frac{\phi^{-3} \phi_{p}}{\left(\phi^{-2}+|\nabla f|^{2}\right)^{3 / 2}}-\frac{f^{n} A_{p n}}{\phi^{-2}+|\nabla f|^{2}}+\frac{f^{n}\left((\log \phi)_{p} f_{n}+(\log \phi)_{n} f_{p}+\phi f^{m} \phi_{m} f_{p} f_{n}\right)}{\left(\phi^{-2}+|\nabla f|^{2}\right)^{3 / 2}} .
\end{aligned}
$$

Therefore

$$
\begin{aligned}
& \left\langle\partial_{t}, N\right\rangle\left(\Gamma_{p n}^{p}-\bar{\Gamma}_{p n}^{p}\right)(\log \phi)^{n}-\left(\partial_{p}\left\langle\partial_{t}, N\right\rangle\right)(\log \phi)^{p} \\
= & -\frac{(\log \phi)^{n} \phi \phi^{m} f_{m} f_{n}}{\left(\phi^{-2}+|\nabla f|^{2}\right)^{1 / 2}}+\frac{(\log \phi)^{p} \phi^{-3} \phi_{p}}{\left(\phi^{-2}+|\nabla f|^{2}\right)^{3 / 2}} \\
& +\frac{(\log \phi)^{p} f^{n}\left[(\log \phi)_{p} f_{n}+(\log \phi)_{n} f_{p}+\phi f^{m} \phi_{m} f_{p} f_{n}\right]}{\left(\phi^{-2}+|\nabla f|^{2}\right)^{3 / 2}} \\
= & \frac{|\nabla \log \phi|^{2}}{\left(\phi^{-2}+|\nabla f|^{2}\right)^{1 / 2}},
\end{aligned}
$$

and (3.6) follows.

The desired identity is now obtained by substituting (3.3), (3.5), and (3.6), into (3.2). 
We are now ready to prove the Harnack inequality.

Theorem 3.2. Suppose that the surface $\Sigma$ satisfies (2.2). Then there exist constants $\rho>0$ and $c$ independent of $\Sigma$, but dependent on $|\log \phi|_{C^{2, \alpha}}$ and $|F|_{C^{1, \alpha}}$, such that

$$
\sup _{\Sigma \cap B_{\rho}^{4}\left(x_{0}\right)}\left\langle\partial_{t},-N\right\rangle \leq c \inf _{\Sigma \cap B_{\rho}^{4}\left(x_{0}\right)}\left\langle\partial_{t},-N\right\rangle
$$

and

$$
\sup _{\Sigma \cap B_{\rho}^{4}\left(x_{0}\right)}\left|\bar{\nabla} \log \left\langle\partial_{t},-N\right\rangle\right| \leq c .
$$

Proof. The estimates of Theorem 2.4 guarantee that equation (3.1) is uniformly elliptic for small $\rho$. Next, we note that the coefficients of (3.1) are bounded. To see this, observe that $R_{N N}$ poses no problem, and $|A|^{2}$ is bounded by Theorem $[2.2$. Moreover

$$
N(H)=\frac{\phi f^{m} \partial_{m} H}{\sqrt{1+\phi^{2}|\nabla f|^{2}}}
$$

so that $N(H)$ is bounded by Corollary 2.3 .

The Harnack inequality (3.7) now follows immediately. Standard elliptic theory [11] also guarantees that

$$
\sup _{B_{\frac{\rho}{2}}}\left|\bar{\nabla}\left\langle\partial_{t}, N\right\rangle\right| \leq \sup _{B_{\rho}}\left|\left\langle\partial_{t}, N\right\rangle\right| .
$$

Combining this with (3.7) yields

$$
\sup _{B_{\frac{\rho}{2}}}\left|\bar{\nabla}\left\langle\partial_{t}, N\right\rangle\right| \leq c \inf _{B_{\rho}}\left|\left\langle\partial_{t}, N\right\rangle\right| \leq c \inf _{B_{\frac{\rho}{2}}}\left|\left\langle\partial_{t}, N\right\rangle\right|,
$$

from which (3.8) follows.

\section{Global $C^{1}$ Bounds and Existence for the Regularized Equation}

Suppose that the boundary of $M$ is an outermost future apparent horizon with one component, that is

$$
\theta_{+}(\partial M)=0
$$

where the null expansion $\theta_{+}$was defined in (1.7). All arguments to follow may be easily extended to the general case in which the boundary is an outermost apparent horizon, with several future and past horizon components. Take a sufficiently large coordinate sphere $\partial_{\infty} M$ in the asymptotically flat end such that

$$
\theta_{+}\left(\partial_{\infty} M\right)>0
$$

Following [1] and [17] we construct an extension $(\widetilde{M}, \widetilde{g}, \widetilde{k})$, of the initial data $(M, g, k)$, having the following properties:

i) $M \subset \widetilde{M}$ with $\left.\widetilde{g}\right|_{M}=g,\left.\widetilde{k}\right|_{M}=k$, and $\partial_{\infty} \widetilde{M}=\partial_{\infty} M$,

ii) $\widetilde{g}$ is smooth across $\partial M$ and $\widetilde{k}$ is $C^{1,1}$ across $\partial M$,

iii) the region $\widetilde{M}-M$ is foliated by surfaces $S_{\sigma}, \sigma \in\left[0, \sigma_{0}\right)$, with $\theta_{+}\left(S_{\sigma}\right)<0, S_{0}=\partial \widetilde{M}$, and $S_{\sigma_{0}}=\partial M$,

iv) for all sufficiently small $\sigma, \theta_{+}\left(S_{\sigma}\right) \leq-\vartheta<0$ and $\widetilde{k} \equiv 0$. 
For convenience, in what follows, we will denote $\widetilde{g}$ by $g$ and $\widetilde{k}$ by $k$. Moreover, we will extend the warping factor $\phi$ to be positive on all of $\widetilde{M}$. These constructions allow for the existence of appropriate barriers, which in turn leads to a solution for the following Dirichlet problem

$$
\begin{gathered}
H\left(f_{\varepsilon}\right)-K\left(f_{\varepsilon}\right)=\varepsilon f_{\varepsilon} \text { on } \widetilde{M} \\
f_{\varepsilon}=\frac{\vartheta}{2 \varepsilon} \quad \text { on } \partial \widetilde{M}, \quad f_{\varepsilon}=0 \quad \text { on } \partial_{\infty} \widetilde{M} .
\end{gathered}
$$

The first step is to obtain global $C^{1}$ bounds. Observe that a direct application of the maximum principle yields the $C^{0}$ bound

$$
\sup _{\widetilde{M}}\left|f_{\varepsilon}\right| \leq c \varepsilon^{-1}
$$

where the constant $c$ depends on $|k|_{C^{0}}$ and $\vartheta$. In order to apply the maximum principle to obtain bounds on first derivatives, we need to establish the boundary gradient estimates. Let $\tau(x)=$ $\operatorname{dist}(x, \partial \widetilde{M})$, and denote by $S_{\tau}$ the level sets of the geodesic flow $\partial_{\tau}=n$ emanating from $\partial \widetilde{M}$, where $n$ is the unit outer normal (pointing towards spatial infinity) of the surfaces $S_{\tau}$. The barrier functions $\psi_{ \pm}(\tau)$ will be functions of $\tau$ alone. In order to find sub and super solutions notice that

$$
\begin{aligned}
& \left(g^{i j}-\frac{\phi^{2} \psi^{i} \psi^{j}}{1+\phi^{2}|\nabla \psi|^{2}}\right)\left(\frac{\phi \nabla_{i j} \psi+\phi_{i} \psi_{j}+\phi_{j} \psi_{i}}{\sqrt{1+\phi^{2}|\nabla \psi|^{2}}}\right) \\
= & \left(1-\frac{\phi^{2} \psi^{\prime 2}}{1+\phi^{2} \psi^{\prime 2}}\right)\left(\frac{\phi \psi^{\prime \prime}+2 \phi^{\prime} \psi^{\prime}}{\sqrt{1+\phi^{2} \psi^{\prime 2}}}\right)+\left(\left.g\right|_{S_{\tau}}\right)^{i j} \frac{\phi \nabla_{i j} \psi}{\sqrt{1+\phi^{2} \psi^{\prime 2}}},
\end{aligned}
$$

and

$$
\left(g^{i j}-\frac{\phi^{2} \psi^{i} \psi^{j}}{1+\phi^{2}|\nabla \psi|^{2}}\right) k_{i j}=\left(1-\frac{\phi^{2} \psi^{\prime 2}}{1+\phi^{2} \psi^{\prime 2}}\right) k_{n n}+\operatorname{Tr}_{S_{\tau}} k
$$

where $\psi^{\prime}=\frac{d \psi}{d \tau}$. Moreover

$$
\nabla_{i j} \psi=\partial_{i j} \psi-\Gamma_{i j}^{m} \partial_{m} \psi=\nabla_{i j}^{S_{\tau}} \psi-\Gamma_{i j}^{n} \partial_{n} \psi=\nabla_{i j}^{S_{\tau}} \psi+A_{i j}^{S_{\tau}} \partial_{n} \psi,
$$

where $\nabla^{S_{\tau}}$ denotes the induced connection on $S_{\tau}$ and $A^{S_{\tau}}$ is its second fundamental form. Therefore

$$
\begin{aligned}
& H(\psi)-K(\psi)-\varepsilon \psi \\
= & \frac{\phi \psi^{\prime \prime}+2 \phi^{\prime} \psi^{\prime}}{\left(1+\phi^{2} \psi^{\prime 2}\right)^{3 / 2}}+\frac{\phi \psi^{\prime} H_{S_{\tau}}}{\sqrt{1+\phi^{2} \psi^{\prime 2}}}-\frac{k_{n n}}{1+\phi^{2} \psi^{\prime 2}}-\operatorname{Tr}_{S_{\tau}} k-\varepsilon \psi \\
= & \frac{\phi \psi^{\prime \prime}+2 \phi^{\prime} \psi^{\prime}}{\left(1+\phi^{2} \psi^{\prime 2}\right)^{3 / 2}}+\frac{\phi \psi^{\prime} \theta_{+}\left(S_{\tau}\right)}{\sqrt{1+\phi^{2} \psi^{\prime 2}}}-\frac{k_{n n}}{1+\phi^{2} \psi^{\prime 2}}-\left(1+\frac{\phi \psi^{\prime}}{\sqrt{1+\phi^{2} \psi^{\prime 2}}}\right) \operatorname{Tr}_{S_{\tau}} k-\varepsilon \psi .
\end{aligned}
$$

Let $\psi_{-}(\tau)=a-b \tau$ where $a$ and $b$ will be chosen appropriately and positive. In order that $\psi_{-}$agree with $f_{\varepsilon}$ at $\partial \widetilde{M}$ we set $a=\frac{\vartheta}{2 \varepsilon}$. Then for $\tau \in\left[0, \tau_{0}\right]$, with $\tau_{0}$ sufficiently small,

$$
H\left(\psi_{-}\right)-K\left(\psi_{-}\right)-\varepsilon \psi_{-} \geq \vartheta-\varepsilon a+\varepsilon b \tau+O\left(b^{-1}\right) \geq \frac{\vartheta}{2}+O\left(b^{-1}\right) \geq 0
$$

if $b$ is chosen large enough depending on $\phi$. Similarly an upper barrier may be constructed in the form $\psi_{+}(\tau)=a+b \tau$. Note that in the case of an upper barrier we use the fact that $k \equiv 0$ near $\partial \widetilde{M}$ to 
deal with the $\operatorname{Tr}_{S_{\tau}} k$ term. Now choose $b$ so large that $a+b \tau_{0} \geq \sup _{\widetilde{M}}\left|f_{\varepsilon}\right|$ and $a-b \tau_{0} \leq-\sup _{\widetilde{M}}\left|f_{\varepsilon}\right|$, then by a standard comparison argument $\psi_{-} \leq f_{\varepsilon} \leq \psi_{+}$for $\tau \in\left[0, \tau_{0}\right]$. It follows that

$$
\sup _{\partial \widetilde{M}}\left|\nabla f_{\varepsilon}\right| \leq b \leq c \varepsilon^{-1} \text {. }
$$

Moreover, a standard barrier construction at $\partial_{\infty} \widetilde{M}$ also yields a boundary gradient estimate.

We now use the Bernstein method to obtain global $C^{1}$ estimates. For convenience we temporarily drop the subscript $\varepsilon$ from $f_{\varepsilon}$. Differentiate equation (4.3) with respect to $\partial_{p}$ to find

$$
\begin{aligned}
& \bar{g}^{i j}\left[\frac{\phi_{p} f_{; i j}+\phi f_{; i j p}+\phi_{; i p} f_{j}+\phi_{i} f_{; j p}+\phi_{; j p} f_{i}+\phi_{j} f_{; i p}}{\sqrt{1+\phi^{2}|\nabla f|^{2}}}\right] \\
& -\bar{g}^{i j}\left[\frac{\left(\phi f_{; i j}+\phi_{i} f_{j}+\phi_{j} f_{i}\right)\left(\phi \phi_{p}|\nabla f|^{2}+\phi^{2} f_{; m p} f^{m}\right)}{\left(1+\phi^{2}|\nabla f|^{2}\right)^{3 / 2}}-k_{i j ; p}\right] \\
& -2\left[\frac{\phi f_{; i j}+\phi_{i} f_{j}+\phi_{j} f_{i}}{\sqrt{1+\phi^{2}|\nabla f|^{2}}}-k_{i j}\right]\left[\frac{\phi \phi_{p} f^{i} f^{j}+\phi^{2} f_{; p} f^{j}}{1+\phi^{2}|\nabla f|^{2}}-\frac{\phi^{2} f^{i} f^{j}\left(\phi \phi_{p}|\nabla f|^{2}+\phi^{2} f_{; m p} f^{m}\right)}{\left(1+\phi^{2}|\nabla f|^{2}\right)^{2}}\right] \\
= & \varepsilon f_{p} .
\end{aligned}
$$

Notice that the Ricci commutation formula yields

$$
\begin{aligned}
\bar{g}^{i j} \phi f^{p} f_{; i j p} & =\bar{g}^{i j} \phi f^{p}\left(f_{; p i j}+R_{i j p}^{m} f_{m}\right) \\
& =\nabla_{j}\left(\bar{g}^{i j} \phi f^{p} f_{; p i}\right)-\bar{g}^{i j} \phi f_{; i}^{p} f_{; p j}-\nabla_{j}\left(\bar{g}^{i j} \phi\right) f^{p} f_{; p i}+\bar{g}^{i j} \phi f^{p} f_{m} R_{i j p}^{m} .
\end{aligned}
$$

Thus, if $u=|\nabla f|^{2}$, then (4.8) implies that

$$
\frac{\nabla_{j}\left(\phi \bar{g}^{i j} u_{i}\right)}{2 \sqrt{1+\phi^{2}|\nabla f|^{2}}}+B^{i} u_{i}+B u^{1 / 2}+\frac{\bar{g}^{i j}\left(f^{p} \phi_{p} f_{; i j}-\phi f_{; i}{ }^{p} f_{; p j}\right)}{\sqrt{1+\phi^{2}|\nabla f|^{2}}} \geq \varepsilon u,
$$

for some coefficients $B^{i}$ and $B$, with $B$ bounded. Moreover at a critical point for $u$,

$$
\bar{g}^{i j}\left(f^{p} \phi_{p} f_{; i j}-\phi f_{; i}^{p} f_{; p j}\right)=g^{i j}\left(f^{p} \phi_{p} f_{; i j}-\phi f_{; i}{ }^{p} f_{; p j}\right) \leq-\frac{1}{2} \phi\left|\nabla^{2} f\right|^{2}+c u .
$$

An alternate approach to deal with the $\bar{g}^{i j} f_{; i j}$ term is to solve for it in (4.3), leaving a manageable number of first derivatives of $f$. Hence from the maximum principle we obtain

$$
\sup _{\widetilde{M}}\left|\nabla f_{\varepsilon}\right| \leq c \varepsilon^{-1} .
$$

With the global $C^{1}$ bounds the equation (4.3) is uniformly elliptic. Standard theory [11 then gives $C^{1, \alpha}$ estimates up to the boundary. The Schauder estimates may now be applied to obtain global $C^{2, \alpha}$ bounds. Thus we may apply the continuity method, as in [1], to the family of equations

$$
\begin{gathered}
H\left(f_{\varrho, \varepsilon}\right)-\varrho K\left(f_{\varrho, \varepsilon}\right)=\varepsilon f_{\varrho, \varepsilon}, \quad 0 \leq \varrho \leq 1, \\
f_{\varrho, \varepsilon}=\frac{\varrho \vartheta}{2 \varepsilon} \quad \text { on } \partial \widetilde{M}, \quad f_{\varrho, \varepsilon}=0 \quad \text { on } \partial_{\infty} \widetilde{M},
\end{gathered}
$$

to obtain a solution of boundary value problem (4.3), (4.4). Furthermore, by sending $\partial_{\infty} \widetilde{M}$ to infinity we obtain a solution on all of $\widetilde{M}$ with the usual decay (1.9). Lastly, note that the global estimates of this section also hold when $M$ does not have boundary; of course they are easier to prove in this case, as there is no need for an extension $\widetilde{M}$ or boundary gradient estimates. Thus, in the case that 
$M$ does not have boundary, we may set $F=\varepsilon f_{\varepsilon}$ and apply the results of Sections 2 and 3 , as well as the global estimates of this section, and let $\varepsilon \rightarrow 0$ to obtain the following result (see [19] for details).

Theorem 4.1. Suppose that $(M, g, k)$ is a smooth, complete, asymptotically flat initial data set, and that $\phi$ is smooth, strictly positive, and satisfies (1.8). Then there exist disjoint open sets $\Omega_{+}, \Omega_{-} \subset M$, and a smooth function $f: M-\left(\Omega_{+} \cup \Omega_{-}\right) \rightarrow \mathbb{R}$ satisfying the generalized Jang equation (1.6) as well as (1.9). Furthermore $\partial \Omega_{+}\left(\partial \Omega_{-}\right)$is a future (past) apparent horizon with $f(x) \rightarrow \pm \infty$ as $x \rightarrow \partial \Omega_{ \pm}$. More precisely graph $(f)$ is asymptotic to the cylinders $\partial \Omega_{+} \times \mathbb{R}_{+}$and $\partial \Omega_{-} \times \mathbb{R}_{-}$.

\section{Blow-Up Solutions for the Generalized Jang Equation}

Consider the solutions $f_{\varepsilon}$ given in the previous section, defined on $\widetilde{M}$ and with fall-off (1.9) at spatial infinity. Our goal in the current section is to produce solutions which blow-up at the outermost apparent horizon boundary of $M$, by letting $\varepsilon \rightarrow 0$. We will also construct appropriate super solutions in order to obtain an estimate for the rate of blow-up. As in the previous section we will assume here that the boundary of $M$ is an outermost future apparent horizon with one component. All arguments to follow may be easily extended to the general case in which the boundary is an outermost apparent horizon, with several future and past horizon components.

Observe that the gradient estimate (4.9) and the boundary condition (4.4) imply that there exists $\kappa$ independent of $\varepsilon$, such that

$$
f_{\varepsilon}(x) \geq \frac{\vartheta}{4 \varepsilon} \quad \text { for } \quad \operatorname{dist}(x, \partial \widetilde{M})<\kappa .
$$

As in [19] a subsequence of the $\varepsilon$-Jang surfaces converges to a properly embedded submanifold of $\widetilde{M} \times \mathbb{R}$. This convergence determines three types of domains:

$$
\begin{aligned}
\widetilde{M}_{+} & =\left\{x \in \widetilde{M} \mid f_{\varepsilon_{i}}(x) \rightarrow+\infty \text { locally uniformly as } i \rightarrow \infty\right\}, \\
\widetilde{M}_{-} & =\left\{x \in \widetilde{M} \mid f_{\varepsilon_{i}}(x) \rightarrow-\infty \text { locally uniformly as } i \rightarrow \infty\right\}, \\
\widetilde{M}_{0} & =\left\{x \in \widetilde{M}\left|\limsup _{i \rightarrow \infty}\right| f_{\varepsilon_{i}}(x) \mid<\infty\right\} .
\end{aligned}
$$

By (5.1), $\widetilde{M}_{+} \neq \emptyset$ and $\widetilde{M}_{+}$contains a neighborhood of $\partial \widetilde{M}$. Since $\partial \widetilde{M}_{+}-\partial \widetilde{M}$ consists of apparent horizons and the region $\widetilde{M}-M$ is foliated by surfaces with $\theta_{+}<0$, we must have that $\widetilde{M}-M \subset \widetilde{M}_{+}$. Thus $\partial \widetilde{M}_{+}$is an apparent horizon in $M$. As $\partial M$ is the outermost apparent horizon in $M$, we conclude that the closure of $\widetilde{M}_{+}$is $\widetilde{M}-M$. A standard barrier argument [19] at spatial infinity shows that the $\varepsilon$-Jang surfaces are uniformly bounded there, so that $\widetilde{M}_{0}$ contains a neighborhood of spatial infinity. It follows that $\widetilde{M}_{0}=M$, and the limiting Jang surface $\Sigma$ blows-up in the form of a cylinder over $\partial M$. This line of argument was used by Metzger [17] for the classical Jang equation.

This result holds for all warping factors $\phi$ which are strictly positive up to and including the boundary. We would now like to examine what happens when $\phi$ is allowed to vanish on the boundary. From now on, let

$$
\tau(x)=\operatorname{dist}(x, \partial M),
$$

the distance to the boundary, and denote by $S_{\tau}$ the level sets of the geodesic flow $\partial_{\tau}=n$ emanating from $\partial M$, where $n$ is the unit outer normal (pointing towards spatial infinity) of the surfaces $S_{\tau}$. We assume that

$$
\phi=\tau^{b} \widetilde{\phi} \quad \text { near } \quad \partial M
$$


where $\widetilde{\phi}$ is some positive function, and then set

$$
\phi_{\delta}=(\tau+\delta)^{b} \widetilde{\phi} \text { near } \partial M,
$$

where $\delta>0$ is a constant. The function $\phi_{\delta}$ is then naturally extended to the rest of $M$. When $\phi$ is replaced by $\phi_{\delta}$, we write the corresponding generalized Jang equation as

$$
H_{\delta}(f)-K_{\delta}(f)=0 .
$$

For each $\delta>0$ a blow-up solution $f_{\delta}$ of (5.3) exists. We aim to show that for some subsequence $\delta_{i} \rightarrow 0$, the surfaces $\Sigma_{\delta_{i}}=\left\{t=f_{\delta_{i}}(x)\right\}$ converge smoothly away from the boundary to a blow-up solution $\Sigma=\{t=f(x)\}$ whose asymptotics at the boundary depend on the rates at which $\phi$ and $\theta_{+}$ vanish.

We now establish the existence of blow-up solutions with the desired upper bound in the first case of Theorem 1.1. The lower bound will be established in the next section.

Proposition 5.1. Suppose that $(M, g, k)$ is a smooth, asymptotically flat initial data set, with outermost apparent horizon boundary $\partial M$ consisting of a single future apparent horizon component. Suppose further that $\phi$ is a smooth function, strictly positive away from $\partial M$ and satisfying (1.8) and (1.10), and that $\theta_{+}$satisfies (1.12). Then for $b \geq-\frac{l-1}{2}$, there exists a smooth solution $f$ of the generalized Jang equation (1.6), satisfying (1.9), and in a neighborhood of $\partial M$

$$
\begin{aligned}
& f \leq \alpha \tau^{-b-\frac{l-1}{2}}+\beta \quad \text { if } \quad b>-\frac{l-1}{2}, \\
& f \leq-\alpha \log \tau+\beta \quad \text { if } \quad b=-\frac{l-1}{2}
\end{aligned}
$$

for some positive constants $\alpha$ and $\beta$.

Proof. Let $f_{\delta, \varepsilon}$ denote solutions of the $\varepsilon$-regularized generalized Jang equation with warping factor $\phi_{\delta}$, as constructed in the previous section,

$$
H_{\delta}\left(f_{\delta, \varepsilon}\right)-K_{\delta}\left(f_{\delta, \varepsilon}\right)=\varepsilon f_{\delta, \varepsilon} .
$$

We now proceed to construct an appropriate upper barrier function $\psi$ for $f_{\delta, \varepsilon}$ which is independent of $\delta$ and $\varepsilon$. For this we will assume that (1.12) holds for $\tau \in\left[0, \tau_{0}\right]$, with $\tau_{0}$ sufficiently small. The upper barrier $\psi$ will be a function of $\tau$ alone, namely $\psi=\psi(\tau)$. Recall that

$$
\begin{gathered}
H_{\delta}(\psi)-K_{\delta}(\psi)-\varepsilon \psi=\frac{\phi_{\delta} \psi^{\prime \prime}+2 \phi_{\delta}^{\prime} \psi^{\prime}}{\left(1+\phi_{\delta}^{2} \psi^{\prime 2}\right)^{3 / 2}}+\frac{\phi_{\delta} \psi^{\prime} \theta_{+}\left(S_{\tau}\right)}{\sqrt{1+\phi_{\delta}^{2} \psi^{\prime 2}}}-\frac{k_{n n}}{1+\phi_{\delta}^{2} \psi^{\prime 2}} \\
-\left(1+\frac{\phi_{\delta} \psi^{\prime}}{\sqrt{1+\phi_{\delta}^{2} \psi^{\prime 2}}}\right) \operatorname{Tr}_{S_{\tau}} k-\varepsilon \psi
\end{gathered}
$$

In the following, $\psi$ will be a decreasing function, that is, $\psi^{\prime}<0$. Hence, we have

$$
\begin{aligned}
H_{\delta}(\psi)-K_{\delta}(\psi)-\varepsilon \psi=- & \theta_{+}\left(S_{\tau}\right)+\frac{\phi_{\delta} \psi^{\prime \prime}+2 \phi_{\delta}^{\prime} \psi^{\prime}}{\left(1+\phi_{\delta}^{2} \psi^{\prime 2}\right)^{3 / 2}}-\frac{k_{n n}}{1+\phi_{\delta}^{2} \psi^{\prime 2}} \\
& +\frac{H_{S_{\tau}}}{\sqrt{1+\phi_{\delta}^{2} \psi^{\prime 2}}\left(\sqrt{1+\phi_{\delta}^{2} \psi^{\prime 2}}-\phi_{\delta} \psi^{\prime}\right)}-\varepsilon \psi .
\end{aligned}
$$

Consider

$$
\psi(\tau)=\alpha \tau^{-a}+\beta
$$


for some constants $a, \alpha, \beta \geq 0$. In order for $\left|\phi \psi^{\prime}\right| \rightarrow \infty$ as $\tau \rightarrow 0$, we require that $a>b-1$. A straightforward calculation shows that

$$
\frac{\left|\phi_{\delta} \psi^{\prime \prime}+2 \phi_{\delta}^{\prime} \psi^{\prime}\right|}{\left(1+\phi_{\delta}^{2} \psi^{\prime 2}\right)^{3 / 2}}+\frac{1}{1+\phi_{\delta}^{2} \psi^{\prime 2}} \leq \frac{C}{a^{2} \alpha^{2}}(a+b+1) \tau^{2 a-2 b+1},
$$

for $\tau$ sufficiently small. By the assumption $\theta_{+} \geq c^{-1} \tau^{l}$, we obtain

$$
H_{\delta}(\psi)-K_{\delta}(\psi)-\varepsilon \psi \leq-c^{-1} \tau^{l}+\frac{C}{a^{2} \alpha^{2}}(a+b+1) \tau^{2 a-2 b+1} .
$$

Now set $a=b+\frac{l-1}{2}$, and notice that this automatically satisfies the previous condition $a>b-1$. If $a>0$ and $\alpha$ is chosen sufficiently large, then $\psi$ is a super solution,

$$
H_{\delta}(\psi)-K_{\delta}(\psi)-\varepsilon \psi \leq 0 .
$$

If $a=0$, then setting

$$
\psi(\tau)=-\alpha \log \tau+\beta
$$

yields a similar result.

In order to obtain the estimate, recall that $\left|f_{\delta, \varepsilon}\right|$ will be uniformly bounded away from the boundary, say at the surface corresponding to $\tau=\tau_{0}>0$. This follows from the Harnack inequality and parametric estimates, as in [19]. We may then choose $\beta$ sufficiently large independent of $\delta$ and $\varepsilon$ such that $\psi\left(\tau_{0}\right) \geq\left. f_{\delta, \varepsilon}\right|_{S_{\tau_{0}}}$. A standard comparison argument then shows that

$$
\left.f_{\delta, \varepsilon}\right|_{S_{\tau}} \leq \psi(\tau) \text { for } \tau \in\left(0, \tau_{0}\right] .
$$

As we have shown above, letting $\varepsilon \rightarrow 0$ yields a blow-up solution $\Sigma_{\delta}$ to the generalized Jang equation for each positive $\delta$. The solution $f_{\delta}$ must of course also satisfy the asymptotics (5.6).

Let us now extract a subsequence $\delta_{i} \rightarrow 0$ such that the surfaces $\Sigma_{\delta_{i}}$ converge smoothly away from the boundary to a solution $\Sigma=\{t=f(x)\}$ of the generalized Jang equation. This may be proved in the usual way [19], making use of the parametric estimates of Section 2 and the Harnack inequality of Section 3. Moreover the asymptotics (5.6) still hold for $f$.

Since the warping factor $\phi$ vanishes at the boundary, we cannot say, without further analysis, precisely how the solution behaves at the boundary. For instance, although the solutions $\Sigma_{\delta_{i}}$ blowup in the form of a cylinder over $\partial M$, it may be the case that as $\delta_{i} \rightarrow 0$ the blow-up solutions become arbitrarily close to $\partial M \times \mathbb{R}$ and eventually (in the limit) coincide or 'stick' to the cylinder at certain points. In order to prevent this, certain inequalities should hold between the vanishing rates of $\phi$ and $\theta_{+}$; this issue is the primary focus of the next section.

It turns out that the blow-up rates of (5.4) are not the only asymptotics for the generalized Jang equations. The next result provides other possibilities for upper barriers.

Proposition 5.2. Suppose that $(M, g, k)$ is a smooth, asymptotically flat initial data set, with outermost apparent horizon boundary $\partial M$ consisting of a single future apparent horizon component. Suppose further that $\phi$ is a smooth function, strictly positive away from $\partial M$ and satisfying (1.8) and (1.10), and that $\theta_{+}$satisfies (1.12). Then for $b \geq 1 / 2$, there exists a smooth solution $f$ of the generalized Jang equation (1.6), satisfying (1.9), and in a neighborhood of $\partial M$

$$
\begin{aligned}
& f \leq \alpha \tau^{1-2 b}+\beta \quad \text { if } \quad b>\frac{1}{2}, \\
& f \leq-\alpha \log \tau+\beta \quad \text { if } \quad b=\frac{1}{2},
\end{aligned}
$$

for some positive constants $\alpha$ and $\beta$. 
Proof. Recall the calculation in (5.5). Our strategy will be to choose the upper barrier function $\psi_{\delta}$, so that the second derivative of $\psi_{\delta}$ will dominate all other terms in (5.5), except $-\theta_{+}\left(S_{\tau}\right)$. To this end, consider

$$
\psi_{\delta}^{\prime \prime}+\frac{2 b}{\tau+\delta} \psi_{\delta}^{\prime}=\lambda \psi_{\delta}^{\prime}
$$

for some $\lambda$ to be determined. It is an easy exercise to show that its solution is given by

$$
\psi_{\delta}(\tau)=\mu_{1} \int_{\tau+\delta}^{1} \frac{e^{\lambda s}}{s^{2 b}} d s+\mu_{2},
$$

for some positive $\mu_{1}$ and $\mu_{2}$ to be determined. It is obvious that $\psi_{\delta}$ is monotonically decreasing. Therefore

$$
\begin{aligned}
\psi_{\delta}^{\prime \prime}+2\left(\log \phi_{\delta}\right)^{\prime} \psi_{\delta}^{\prime} & =\psi_{\delta}^{\prime \prime}+\frac{2 b}{\tau+\delta} \psi_{\delta}^{\prime}+2(\log \widetilde{\phi})^{\prime} \psi_{\delta}^{\prime} \leq \psi_{\delta}^{\prime \prime}+\frac{2 b}{\tau+\delta} \psi_{\delta}^{\prime}-c \psi_{\delta}^{\prime} \\
& =(\lambda-c) \psi_{\delta}^{\prime} \leq \frac{\lambda}{2} \psi_{\delta}^{\prime}<0
\end{aligned}
$$

for large $\lambda$. Hence, (5.5) becomes

$$
\begin{aligned}
H_{\delta}\left(\psi_{\delta}\right)-K_{\delta}\left(\psi_{\delta}\right)-\varepsilon \psi_{\delta} & \leq-\theta_{+}+\frac{\lambda \phi_{\delta} \psi_{\delta}^{\prime}}{2\left(1+\phi_{\delta}^{2} \psi_{\delta}^{\prime 2}\right)^{3 / 2}}+c \phi_{\delta}^{-2}\left|\psi_{\delta}^{\prime}\right|^{-2} \\
& \leq-c^{-1} \tau^{l}-\left(\frac{\lambda}{2}-c\right) \phi_{\delta}^{-2}\left|\psi_{\delta}^{\prime}\right|^{-2}<0
\end{aligned}
$$

if again $\lambda$ is large enough. It follows that $\psi_{\delta}$ is a super solution.

Recall that $\left.f_{\delta, \varepsilon}\right|_{\partial M} \leq c \varepsilon^{-1}$ by (4.5), and note that

$$
\begin{aligned}
& \psi_{\delta}(0) \geq \alpha \delta^{1-2 b}+\beta \quad \text { if } \quad b>\frac{1}{2}, \\
& \psi_{\delta}(0) \geq-\alpha \log \delta+\beta \quad \text { if } \quad b=\frac{1}{2} .
\end{aligned}
$$

This shows that $\left.\psi_{\delta}\right|_{\partial M} \geq\left. f_{\delta, \varepsilon}\right|_{\partial M}$ for sufficiently large $\alpha$, if $\delta=\varepsilon^{(2 b-1)^{-1}}$ when $b>1 / 2$, and $\delta=e^{-\varepsilon^{-1}}$ when $b=1 / 2$. The maximum principle then implies (if $\beta$ is chosen large enough) that $f_{\delta, \varepsilon} \leq \psi_{\delta}$ for $\tau \in\left[0, \tau_{0}\right]$. Let us now extract a subsequence $\varepsilon_{i} \rightarrow 0$ such that the surfaces $\Sigma_{\delta_{i}, \varepsilon_{i}}$ converge smoothly away from the boundary to a solution $\Sigma=\{t=f(x)\}$ of the generalized Jang equation. This may be proved in the usual way as at the end of Proposition 5.1, even though here $\delta_{i}=\varepsilon_{i}^{(2 b-1)^{-1}}$ when $b>1 / 2$, and $\delta_{i}=e^{-\varepsilon_{i}^{-1}}$ when $b=1 / 2$. It follows that the solution $f$ must satisfy the asymptotics (5.7).

Again, because the warping factor $\phi$ vanishes at the boundary, we cannot say, without further analysis, precisely how the solution behaves at the boundary. It might coincide or 'stick' to the cylinder over the boundary at certain points. Nevertheless, the barrier construction yields an upper bound for the asymptotics.

We may now compare the different asymptotics (5.4) and (5.7). Observe that $2 b-1<b+\frac{l-1}{2}$ only when $b<\frac{l+1}{2}$, and therefore the asymptotics in (5.7) only improve the ones in (5.4) when $\frac{1}{2} \leq b<\frac{l+1}{2}$. 


\section{Further Analysis of the Blow-Up Solutions}

The purpose of this section is to obtain subsolutions for the generalized Jang equation and to prove Theorem 1.1. It will be shown that subsolutions exist with the same asymptotics as described in Propositions 5.1 and 5.2. An appropriate comparison argument will then be employed to prove Theorem 1.1. As in the previous two sections, we will assume for simplicity that the boundary of $M$ is an outermost future apparent horizon with one component. All arguments to follow may be extended to the general case in which the boundary is an outermost apparent horizon, with several future and past horizon components. Moreover the additional arguments needed for such an extension will be recorded at the end of this section in the proof of Theorem 1.1 .

Let $\psi(\tau)$ be a function of $\tau$ alone, which satisfies $\psi^{\prime}<0$ and

$$
\left|\phi \psi^{\prime}\right| \rightarrow \infty \quad \text { as } \quad \tau \rightarrow 0
$$

Then according to (5.5) there exist bounded functions $c_{1}$ and $c_{2}$, with $c_{1} \geq \frac{1}{2}$ for small $\tau$, such that

$$
H(\psi)-K(\psi)=-\theta_{+}\left(S_{\tau}\right)-\frac{c_{1}}{\tau^{2 b} \psi^{\prime 2}}\left(\frac{\psi^{\prime \prime}}{\psi^{\prime}}+\frac{2 b}{\tau}\right)+\frac{c_{2}}{\tau^{2 b} \psi^{\prime 2}} .
$$

Lemma 6.1. (1) Suppose that $-\frac{l-1}{2} \leq b<\frac{l+1}{2}$, and that (1.12) and (1.10) hold for $\tau \in\left[0, \tau_{0}\right]$. Then for sufficiently small $\tau_{0}$ there exist sub and supersolutions $\underline{\psi}$ and $\bar{\psi}$ of the generalized Jang equation, satisfying (6.1), and such that

$$
\begin{gathered}
\alpha^{-1} \tau^{-b-\frac{l-1}{2}}+\beta^{-1} \leq \underline{\psi}, \bar{\psi} \leq \alpha \tau^{-b-\frac{l-1}{2}}+\beta \quad \text { if } \quad-\frac{l-1}{2}<b<\frac{l+1}{2}, \\
-\alpha^{-1} \log \tau+\beta^{-1} \leq \underline{\psi}, \bar{\psi} \leq-\alpha \log \tau+\beta \quad \text { if } \quad b=-\frac{l-1}{2},
\end{gathered}
$$

for some positive constants $\alpha$ and $\beta$. Moreover

$$
H(\underline{\psi})-K(\underline{\psi}) \geq \lambda \tau^{l}, \quad H(\bar{\psi})-K(\bar{\psi}) \leq-\frac{c^{-1}}{2} \tau^{l},
$$

where the constant $\lambda>0$ may be chosen arbitrarily large and $c^{-1}$ is as in (1.12).

(2) Suppose that $\frac{1}{2} \leq b<\frac{l+1}{2}$, and that (1.10) and (1.11) hold for $\tau \in\left[0, \tau_{0}\right]$. Then for sufficiently small $\tau_{0}$ there exist sub and supersolutions $\underline{\chi}$ and $\bar{\chi}$ of the generalized Jang equation, satisfying (6.1), and such that

$$
\begin{aligned}
& \alpha^{-1} \tau^{1-2 b}+\beta^{-1} \leq \underline{\chi}, \bar{\chi} \leq \alpha \tau^{1-2 b}+\beta \quad \text { if } \quad \frac{1}{2}<b<\frac{l+1}{2}, \\
& -\alpha^{-1} \log \tau+\beta^{-1} \leq \underline{\chi}, \bar{\chi} \leq-\alpha \log \tau+\beta \quad \text { if } \quad b=\frac{1}{2},
\end{aligned}
$$

for some positive constants $\alpha$ and $\beta$. Moreover

$$
H(\underline{\chi})-K(\underline{\chi}) \geq \lambda \tau^{\widetilde{l}}, \quad H(\bar{\chi})-K(\bar{\chi}) \leq-\lambda \tau^{\widetilde{l}},
$$

where the constant $\lambda>0$ may be chosen arbitrarily large and $2 b-1<\widetilde{l} \leq \min (l, 2 b)$.

Proof. (1) First consider the case $-\frac{l-1}{2}<b<\frac{l+1}{2}$ and take the most obvious choice

$$
\psi(\tau)=\alpha \tau^{-a}+\beta,
$$

where $a$ is to be determined. A calculation shows that

$$
\frac{\psi^{\prime \prime}}{\psi^{\prime}}+\frac{2 b}{\tau}=\frac{2 b-a-1}{\tau}
$$


and $\left|\phi \psi^{\prime}\right|=a \alpha \tau^{b-a-1} \rightarrow \infty$ as $\tau \rightarrow 0$ if $a>b-1$. By applying (1.12) to (6.2) we then have

$$
H(\psi)-K(\psi)=-c_{3} \tau^{l}-\frac{(2 b-a-1) c_{1}}{a^{2} \alpha^{2}} \tau^{2 a-2 b+1}+\frac{c_{2}}{a^{2} \alpha^{2}} \tau^{2 a-2 b+2},
$$

where $c_{3} \geq c^{-1}>0$ is a bounded function. Setting $a=b+\frac{l-1}{2}$ yields

$$
H(\psi)-K(\psi)=-c_{3} \tau^{l}+\frac{2(l+1-2 b) c_{1}}{\alpha^{2}(2 b+l-1)^{2}} \tau^{l}+\frac{4 c_{2}}{\alpha^{2}(2 b+l-1)^{2}} \tau^{l+1} .
$$

Thus if $\tau_{0}$ is sufficiently small, then by defining $\underline{\psi}$ to have the structure of $\psi$ with $\alpha$ sufficiently small

$$
H(\underline{\psi})-K(\underline{\psi}) \geq \alpha^{-1} \tau^{l},
$$

and by defining $\bar{\psi}$ to have the structure of $\psi$ with $\alpha$ sufficiently large

$$
H(\bar{\psi})-K(\bar{\psi}) \leq-\frac{c^{-1}}{2} \tau^{l} .
$$

Now consider the case when $b=-\frac{l-1}{2}$, and take

$$
\psi(\tau)=-\alpha \log \tau+\beta
$$

Observe that

$$
\frac{\psi^{\prime \prime}}{\psi^{\prime}}+\frac{2 b}{\tau}=-\frac{l}{\tau}
$$

and $\left|\phi \psi^{\prime}\right|=\alpha \tau^{-\frac{l+1}{2}} \rightarrow \infty$ as $\tau \rightarrow 0$. By applying (1.12) to (6.2) we then have

$$
H(\psi)-K(\psi)=-c_{3} \tau^{l}+\frac{l c_{1}}{\alpha^{2}} \tau^{l}+\frac{c_{2}}{\alpha^{2}} \tau^{l+1} .
$$

Thus if $\tau_{0}$ is sufficiently small, then by defining $\underline{\psi}$ to have the structure of $\psi$ with $\alpha$ sufficiently small

$$
H(\underline{\psi})-K(\underline{\psi}) \geq \alpha^{-1} \tau^{l}
$$

and by defining $\bar{\psi}$ to have the structure of $\psi$ with $\alpha$ sufficiently large

$$
H(\bar{\psi})-K(\bar{\psi}) \leq-\frac{c^{-1}}{2} \tau^{l} .
$$

(2) Assume that $\frac{1}{2} \leq b<\frac{l+1}{2}$, and let $\chi(\tau)$ be a function of $\tau$ alone satisfying (6.1). According to (6.2) and (1.11)

$$
H(\chi)-K(\chi)=c_{4} \tau^{l}-\frac{c_{1}}{\tau^{2 b} \chi^{\prime 2}}\left(\frac{\chi^{\prime \prime}}{\chi^{\prime}}+\frac{2 b}{\tau}\right)+\frac{c_{2}}{\tau^{2 b} \chi^{\prime 2}}
$$

where $c_{4}$ is a bounded function. This suggests that we study the ODE

$$
\frac{1}{\tau^{2 b} \chi^{\prime 2}}\left(\frac{\chi^{\prime \prime}}{\chi^{\prime}}+\frac{2 b}{\tau}\right)=\mp \lambda \tau^{\widetilde{l}}
$$

The choice of $\mp \lambda$ will be used when defining the sub and supersolutions, respectively. In order to solve (6.4) observe that this equation is equivalent to

$$
\left(\frac{\tau^{-4 b}}{\chi^{\prime 2}}\right)^{\prime}= \pm 2 \lambda \tau^{\widetilde{l}-2 b},
$$

and hence

$$
\chi^{\prime}=-\left(\frac{ \pm 2 \lambda}{\widetilde{l}+1-2 b} \tau^{\widetilde{l}+1+2 b}+\Lambda^{2} \tau^{4 b}\right)^{-\frac{1}{2}}
$$


for some constant $\Lambda$. We choose $\Lambda>0$, since if $\Lambda=0$ and $-\lambda$ is chosen in (6.4), then (6.5) yields similar asymptotics as in (1). Notice also that the expression inside the square root is positive for small $\tau$, since $4 b<\widetilde{l}+1+2 b$ and $\Lambda>0$. It follows that

$$
\frac{\Lambda^{-1}}{2} \tau^{-2 b} \leq-\chi^{\prime} \leq 2 \Lambda^{-1} \tau^{-2 b}
$$

for sufficiently small $\tau$. This shows that (6.1) holds. Furthermore

$$
H(\chi)-K(\chi)=c_{4} \tau^{l} \pm c_{1} \lambda \tau^{\widetilde{l}}+c_{5} \Lambda^{2} \tau^{2 b}
$$

for some bounded function $c_{5}$. We define $\underline{\chi}, \bar{\chi}$ to be the solutions of (6.4) constructed above and corresponding to $-\lambda,+\lambda$ respectively. By choosing $\lambda$ sufficiently large the desired result follows, since $\widetilde{l} \leq \min (l, 2 b)$.

The existence of two subsolutions with different asymptotics, when $\frac{1}{2} \leq b<\frac{l+1}{2}$, indicates that there will be two different blow-up solutions of the generalized Jang equation, one corresponding to each of the distinct asymptotics. These solutions will arise from two different sequences of solutions to the regularized equation. More precisely, consider the generalized Jang equation with $\phi=\tau^{b} \widetilde{\phi}$ replaced by $\phi_{\delta}=(\tau+\delta)^{b} \widetilde{\phi}$, as in (5.3). According to the proof of Proposition [5.1, for each $\delta>0$, there exists a blow-up solution $t=f_{\delta}$ which asymptotically approaches the cylinder $\partial M \times \mathbb{R}$ at a rate given by (5.4). A subsequence will then converge to a blow-up solution of the generalized Jang equation as $\delta \rightarrow 0$, and this solution will satisfy the asymptotics (1.13). The second sequence of solutions will arise from the $\delta$-regularized generalized Jang equation, and will be constructed to have finite values at $\partial M$. However, as $\delta \rightarrow 0$ these boundary values will become arbitrarily large, and a subsequence will converge to a blow-up solution of the generalized Jang equation which satisfies the asymptotics (1.14).

We first analyze the case of the solutions $t=f_{\delta}$ with asymptotics given by (5.4). Consider the $\delta$-regularized generalized Jang equation applied to a function $\psi(\tau)$ as in (6.2),

$$
H_{\delta}(\psi)-K_{\delta}(\psi)=-\theta_{+}\left(S_{\tau}\right)-\frac{c_{1}}{(\tau+\delta)^{2 b} \psi^{\prime 2}}\left(\frac{\psi^{\prime \prime}}{\psi^{\prime}}+\frac{2 b}{\tau+\delta}\right)+\frac{c_{2}}{(\tau+\delta)^{2 b} \psi^{\prime 2}} .
$$

Let $\underline{\psi}$ be the subsolution constructed in (1) of Lemma 6.1, and consider

$$
\underline{\psi}_{\delta}(\tau):=\underline{\psi}(\tau+\delta)
$$

It is clear from the proof of Lemma 6.1 that $\underline{\psi}_{\delta}$ is a subsolution of the $\delta$-regularized generalized Jang equation,

$$
H_{\delta}\left(\underline{\psi}_{\delta}\right)-K_{\delta}\left(\underline{\psi}_{\delta}\right) \geq \lambda(\tau+\delta)^{l}>0
$$

We will show that $\underline{\psi}_{\delta}$ acts as a lower barrier for the regularized solutions $f_{\delta}$.

Proposition 6.2. If $\left.f_{\delta}\right|_{S_{\tau_{0}}} \geq \underline{\psi}_{\delta}\left(\tau_{0}\right)$ then $f_{\delta} \geq \underline{\psi}_{\delta}$ for all $\tau \in\left[0, \tau_{0}\right]$.

Proof. Consider the function $w_{\delta}=f_{\delta}-\underline{\psi}_{\delta}$. Since $\underline{\psi}_{\delta}(0)$ is finite and $f_{\delta}$ blows-up at $\partial M$, we have that $\left.w_{\delta}\right|_{\partial M} \geq 0$; moreover, by assumption $\left.w_{\delta}\right|_{S_{\tau_{0}}} \geq 0$. Suppose that $w_{\delta}$ attains an interior negative minimum, then at that point

It follows that at the minimum point

$$
\nabla f_{\delta}=\nabla \underline{\psi}_{\delta}, \quad \nabla^{2} w_{\delta} \geq 0
$$

$$
\left(H_{\delta}\left(f_{\delta}\right)-K_{\delta}\left(f_{\delta}\right)\right)-\left(H_{\delta}\left(\underline{\psi}_{\delta}\right)-K_{\delta}\left(\underline{\psi}_{\delta}\right)\right)=\left(g^{i j}-\frac{\phi_{\delta}^{2} f_{\delta}^{i} f_{\delta}^{j}}{1+\phi_{\delta}^{2}\left|\nabla f_{\delta}\right|^{2}}\right) \frac{\phi_{\delta} \nabla_{i j} w_{\delta}}{\sqrt{1+\phi_{\delta}^{2}\left|\nabla f_{\delta}\right|^{2}}} \geq 0 .
$$


On the other hand, by (6.7)

$$
\left(H_{\delta}\left(f_{\delta}\right)-K_{\delta}\left(f_{\delta}\right)\right)-\left(H_{\delta}\left(\underline{\psi}_{\delta}\right)-K_{\delta}\left(\underline{\psi}_{\delta}\right)\right) \leq-\lambda(\tau+\delta)^{l}<0
$$

This contradiction shows that $w_{\delta} \geq 0$ for all $\tau \in\left[0, \tau_{0}\right]$.

We are now ready to prove the primary existence result for the first asymptotics of Theorem 1.1 .

Theorem 6.3. Suppose that $(M, g, k)$ is a smooth, asymptotically flat initial data set, with outermost apparent horizon boundary $\partial M$ consisting of a single future apparent horizon component. Suppose further that $\phi$ is a smooth function, strictly positive away from $\partial M$ and satisfying (1.8) and (1.10), and that $\theta_{+}$satisfies (1.12) for $\tau \in\left[0, \tau_{0}\right]$. If $-\frac{l-1}{2} \leq b<\frac{l+1}{2}$ then there exists a smooth solution $f$ of the generalized Jang equation (1.6), satisfying (1.9), and such that the following estimates hold in a neighborhood of $\partial M$

$$
\begin{gathered}
\alpha^{-1} \tau^{-b-\frac{l-1}{2}}+\beta^{-1} \leq f \leq \alpha \tau^{-b-\frac{l-1}{2}}+\beta \quad \text { if } \quad-\frac{l-1}{2}<b<\frac{l+1}{2}, \\
-\alpha^{-1} \log \tau+\beta^{-1} \leq f \leq-\alpha \log \tau+\beta \quad \text { if } \quad b=-\frac{l-1}{2}
\end{gathered}
$$

for some positive constants $\alpha$ and $\beta$.

Proof. According to the proof of Proposition [5.1, for each $\delta>0$, there exists a blow-up solution $t=f_{\delta}$ of equation (1.6) with $\phi$ replaced by $\phi_{\delta}$, and this solution asymptotically approaches the cylinder $\partial M \times \mathbb{R}$ at a rate given by (5.4). As in the proof of Proposition 5.1, there is a subsequence $\delta_{i} \rightarrow 0$ such that these graphs converge smoothly away from the boundary to a solution $t=f$ of the generalized Jang equation (1.6), which satisfies (1.9). This implies that there exists a fixed value for $\underline{\psi}_{\delta_{i}}\left(\tau_{0}\right)$, independent of $\delta_{i}$, such that $\left.f_{\delta_{i}}\right|_{S_{\tau_{0}}} \geq \underline{\psi}_{\delta_{i}}\left(\tau_{0}\right)$. In fact by writing

$$
\underline{\psi}_{\delta_{i}}(\tau)=\alpha_{i}^{-1}\left(\tau+\delta_{i}\right)^{-b-\frac{l-1}{2}}+\beta_{i}^{-1}
$$

we can achieve this by choosing fixed $\alpha_{i}=\alpha$ and $\beta_{i}=\beta$ for all $i$. Therefore $\underline{\psi}_{\delta_{i}}$ converges to a function $\underline{\psi}$ satisfying the estimates (6.3). Moreover by Proposition $\left[6.2, f_{\delta_{i}} \geq \underline{\psi}_{\delta_{i}}\right.$ for $\tau \in\left[0, \tau_{0}\right]$, and therefore $f$ satisfies (6.8).

Blow-up solutions of the generalized Jang equation satisfying (1.14) will now be constructed. These will arise from a sequence of solutions to the $\delta$-regularized equation with finite boundary values. Assume that $\frac{1}{2} \leq b<\frac{l+1}{2}$ and that (1.11) holds, and let $\underline{\chi}$, $\bar{\chi}$ be the functions constructed in Lemma 6.1. Set

$$
\underline{\chi}_{\delta}(\tau):=\underline{\chi}(\tau+\delta) \text { and } \bar{\chi}_{\delta}(\tau):=\bar{\chi}(\tau+\delta),
$$

and note that these translated functions are sub and supersolutions for the $\delta$-regularized generalized Jang equation. In particular, from (6.6) and the proof of Lemma 6.1 we have

$$
H_{\delta}\left(\underline{\chi}_{\delta}\right)-K_{\delta}\left(\underline{\chi}_{\delta}\right) \geq \lambda(\tau+\delta)^{\tilde{l}}, \quad H_{\delta}\left(\bar{\chi}_{\delta}\right)-K_{\delta}\left(\bar{\chi}_{\delta}\right) \leq-\lambda(\tau+\delta)^{\widetilde{l}} .
$$

Let $T_{\delta}=\delta^{1-2 b}$ if $b>\frac{1}{2}$ and $T_{\delta}=-\log \delta$ if $b=\frac{1}{2}$, and observe that $T_{\delta} \sim \underline{\chi}_{\delta}(0) \sim \bar{\chi}_{\delta}(0)$. Consider now the following boundary value problem for the $\delta$-regularized generalized Jang equation

$$
\begin{aligned}
& H_{\delta}\left(h_{\delta}\right)-K_{\delta}\left(h_{\delta}\right)=0 \quad \text { on } \quad M, \\
& h_{\delta}=T_{\delta} \quad \text { on } \quad \partial M, \quad h_{\delta} \rightarrow 0 \quad \text { as } \quad|x| \rightarrow \infty .
\end{aligned}
$$


Proposition 6.4. Suppose that $\partial M$ is an outermost future apparent horizon having one component, and that (1.11) holds for $\tau \in\left[0, \tau_{0}\right]$. If $\frac{1}{2} \leq b<\frac{l+1}{2}$, then there exists a smooth solution of (6.10), (6.11), which is $C^{0}$ up to the boundary, and satisfies the following estimates in a neighborhood of $\partial M$

$$
\begin{aligned}
& \alpha^{-1}(\tau+\delta)^{1-2 b}+\beta^{-1} \leq h_{\delta} \leq \alpha(\tau+\delta)^{1-2 b}+\beta \quad \text { if } \quad \frac{1}{2}<b<\frac{l+1}{2} \\
& -\alpha^{-1} \log (\tau+\delta)+\beta^{-1} \leq h_{\delta} \leq-\alpha \log (\tau+\delta)+\beta \quad \text { if } \quad b=\frac{1}{2}
\end{aligned}
$$

for some positive constants $\alpha$ and $\beta$ independent of $\delta$.

Proof. We first solve a boundary value problem for the $\varepsilon \delta$-regularized generalized Jang equation:

$$
\begin{gathered}
H_{\delta}\left(h_{\delta, \varepsilon}\right)-K_{\delta}\left(h_{\delta, \varepsilon}\right)=\varepsilon\left(h_{\delta, \varepsilon}-\chi\right) \quad \text { on } M, \\
h_{\delta, \varepsilon}=T_{\delta} \quad \text { on } \partial M, \quad h_{\delta, \varepsilon} \rightarrow 0 \quad \text { as } \quad|x| \rightarrow \infty,
\end{gathered}
$$

where $\chi$ is a smooth function on $M$ such that $\chi=T_{\delta}$ on $\partial M, \sup _{M}|\chi| \leq T_{\delta}$, and $\chi \equiv 0$ in a neighborhood of spatial infinity. Moreover, we require that $\chi$ is decreasing in $\tau$ near $\partial M$.

We now employ the continuity method to show that a unique solution exists for (6.13) and (6.14). This is similar to arguments used for (4.10) and (4.11). To begin, observe that the maximum principle yields the bound

$$
\sup _{M}\left|h_{\delta, \varepsilon}\right| \leq T_{\delta}+c \varepsilon^{-1} .
$$

To establish boundary gradient estimates, we construct subsolutions and supersolutions.

Fix a $\tau_{0}$ sufficiently small. For subsolutions, take $\underline{\chi}_{\delta, \varepsilon}$ as in Lemma 6.1. Specifically, $\underline{\chi}_{\delta, \varepsilon}$ satisfies

$$
H_{\delta}\left(\underline{\chi}_{\delta, \varepsilon}\right)-K_{\delta}\left(\underline{\chi}_{\delta, \varepsilon}\right) \geq \lambda(\tau+\delta)^{\tilde{l}}
$$

for some positive $\lambda$. Since this subsolution is a solution to a second order ODE, there are two free parameters that may be chosen appropriately so that

$$
\underline{\chi}_{\delta, \varepsilon}(0)=T_{\delta}, \quad \underline{\chi}_{\delta, \varepsilon}\left(\tau_{0}\right)=-\left(T_{\delta}+c \varepsilon^{-1}\right),
$$

and

$$
\underline{\chi}_{\delta, \varepsilon} \leq \chi \quad \text { for } \quad \tau \in\left[0, \tau_{0}\right]
$$

This shows that $w_{\delta, \varepsilon}:=h_{\delta, \varepsilon}-\underline{\chi}_{\delta, \varepsilon} \geq 0$ along the surfaces $\partial M$ and $S_{\tau_{0}}$. Suppose that $w_{\delta, \varepsilon}$ achieves an interior negative minimum. Then at that point, a standard comparison argument yields

$$
\left[H_{\delta}\left(h_{\delta, \varepsilon}\right)-K_{\delta}\left(h_{\delta, \varepsilon}\right)\right]-\left[H_{\delta}\left(\underline{\chi}_{\delta, \varepsilon}\right)-K_{\delta}\left(\underline{\chi}_{\delta, \varepsilon}\right)\right] \geq 0 .
$$

On the other hand, according to (6.9) and $w_{\delta, \varepsilon}<0$ at the point in question, we have

$$
\begin{aligned}
{\left[H_{\delta}\left(h_{\delta, \varepsilon}\right)-K_{\delta}\left(h_{\delta, \varepsilon}\right)\right]-\left[H_{\delta}\left(\underline{\chi}_{\delta, \varepsilon}\right)-K_{\delta}\left(\underline{\chi}_{\delta, \varepsilon}\right)\right] } & \leq \varepsilon\left(h_{\delta, \varepsilon}-\chi\right)-\lambda(\tau+\delta)^{\widetilde{l}} \\
& =\varepsilon w_{\delta, \varepsilon}+\varepsilon\left(\underline{\chi}_{\delta, \varepsilon}-\chi\right)-\lambda(\tau+\delta)^{\widetilde{l}}<0 .
\end{aligned}
$$

It follows that $h_{\delta, \varepsilon} \geq \underline{\chi}_{\delta, \varepsilon}$ for all $\tau \in\left[0, \tau_{0}\right]$, and this yields a lower bound for $\partial_{\tau} h_{\delta, \varepsilon}$.

For supersolutions, let $\tilde{\chi}$ be defined in the same way as $\bar{\chi}$, from the proof of Lemma 6.1, except that (6.5) is replaced by

$$
\widetilde{\chi}^{\prime}=\left(\frac{-2 \lambda}{\widetilde{l}+1-2 b} \tau^{\widetilde{l}+1+2 b}+\Lambda^{2} \tau^{4 b}\right)^{-\frac{1}{2}}
$$


Then $\tilde{\chi}_{\delta, \varepsilon}:=\tilde{\chi}(\tau+\delta)$ satisfies

$$
H_{\delta}\left(\widetilde{\chi}_{\delta, \varepsilon}\right)-K_{\delta}\left(\widetilde{\chi}_{\delta, \varepsilon}\right) \leq-\lambda(\tau+\delta)^{\widetilde{l}}
$$

Choose the two parameters defining $\tilde{\chi}_{\delta, \varepsilon}$ appropriately so that

$$
\tilde{\chi}_{\delta, \varepsilon}(0)=T_{\delta}, \quad \tilde{\chi}_{\delta, \varepsilon}\left(\tau_{0}\right)=T_{\delta}+c \varepsilon^{-1} .
$$

Then $\bar{\chi}_{\delta, \varepsilon} \geq \chi$ for all $\tau \in\left[0, \tau_{0}\right]$. As above, a comparison argument can be employed to show that $h_{\delta, \varepsilon} \leq \widetilde{\chi}_{\delta, \varepsilon}$ for all $\tau \in\left[0, \tau_{0}\right]$. This yields an upper bound for $\partial_{\tau} h_{\delta, \varepsilon}$. A solution of (6.13), (6.14) is now guaranteed, and uniqueness follows from a maximum principle argument.

As in the proof of Theorem 6.3, we may now let $\varepsilon \rightarrow 0$ and extract a subsequence of solutions, still denoted $h_{\delta, \varepsilon}$, which converges smoothly away from the horizon to a solution $h_{\delta}$ of (6.10). Moveover

$$
h_{\delta} \rightarrow 0 \quad \text { as } \quad|x| \rightarrow \infty .
$$

We need to show that

$$
h_{\delta}=T_{\delta} \quad \text { on } \quad \partial M .
$$

Note that the functions $h_{\delta, \varepsilon}$ are uniformly bounded, independent of $\varepsilon$ (in fact independent of $\delta$ as well), when restricted to the surface $S_{\tau_{0}}$. It follows that $\left.h_{\delta, \varepsilon}\right|_{S_{\tau_{0}}}<T_{\delta}$ for $\delta$ sufficiently small. We may then choose sub and supersolutions $\underline{\chi}_{\delta} \leq \bar{\chi}_{\delta}$, satisfying (6.9), the estimate (6.12) with constants $\alpha$ and $\beta$ independent of $\varepsilon$, and such that

$$
\underline{\chi}_{\delta}(0)=\left.h_{\delta, \varepsilon}\right|_{\partial M}=\bar{\chi}_{\delta}(0), \quad \underline{\chi}_{\delta}\left(\tau_{0}\right) \leq\left. h_{\delta, \varepsilon}\right|_{S_{\tau_{0}}} \leq \bar{\chi}_{\delta}\left(\tau_{0}\right),
$$

and

$$
\underline{\chi}_{\delta} \leq \chi \leq \bar{\chi}_{\delta} \quad \text { for } \quad \tau \in\left[0, \tau_{0}\right]
$$

A comparison argument, as in the first half of this proof, may now be used to establish (6.12) for $h_{\delta, \varepsilon}$. This establishes $C^{0}$ and boundary gradient estimates, independent of $\varepsilon$. We may now apply Theorem 15.2 of [1] to obtain global $C^{1}$ bounds independent of $\varepsilon$. It follows that the limit $h_{\delta}$ is $C^{0}$ up to the boundary.

Lastly we may take the subsequential limit $h_{\delta} \rightarrow h$ as $\delta \rightarrow 0$, to find that the functions $h_{\delta}$ are uniformly bounded, independent of $\delta$, when restricted to the surface $S_{\tau_{0}}$. Then as in the previous paragraph, sub and supersolutions $\underline{\chi}_{\delta}$ and $\bar{\chi}_{\delta}$ may be used to obtain the estimate (6.12) with constants $\alpha$ and $\beta$ independent of $\delta$.

Theorem 6.5. Suppose that $(M, g, k)$ is a smooth, asymptotically flat initial data set, with outermost apparent horizon boundary $\partial M$ consisting of a single future apparent horizon component. Suppose further that $\phi$ is a smooth function, strictly positive away from $\partial M$ and satisfying (1.8) and (1.10), and that $\theta_{+}$satisfies (1.11). If $\frac{1}{2} \leq b<\frac{l+1}{2}$ then there exists a smooth solution $h$ of the generalized Jang equation (1.6), satisfying (1.9), and such that the following estimates hold in a neighborhood of $\partial M$

$$
\begin{gathered}
\alpha^{-1} \tau^{1-2 b}+\beta^{-1} \leq h \leq \alpha \tau^{1-2 b}+\beta \quad \text { if } \quad \frac{1}{2}<b<\frac{l+1}{2}, \\
-\alpha^{-1} \log \tau+\beta^{-1} \leq h \leq-\alpha \log \tau+\beta \quad \text { if } \quad b=\frac{1}{2},
\end{gathered}
$$

for some positive constants $\alpha$ and $\beta$. 
Proof. By Proposition 6.4, for each $\delta>0$ there exists a solution $t=h_{\delta}$ of boundary value problem (6.10), (6.11). As in the proof of Theorem 6.3, there is a subsequence $\delta_{i} \rightarrow 0$ such that these graphs converge smoothly away from the boundary to a solution $t=h$ of the generalized Jang equation (1.6), which satisfies (1.9). Since the constants $\alpha$ and $\beta$ in (6.12) are independent of $\delta$, the limit $h$ satisfies (6.15).

Proof of Theorem 1.1. This follows from Theorems6.3 and 6.5 if the boundary has one future apparent horizon component. Similar arguments hold if the boundary has a single past apparent horizon component.

Now consider the case of multiple components in which some components belong to the category (1) and some belong to the category (2). The asymptotics of (1) arise from taking the limit as $\varepsilon \rightarrow 0$ to obtain a blow-up solution, and then taking the limit as $\delta \rightarrow 0$. The asymptotics of (2) arise from taking the limit as $\varepsilon \rightarrow 0$ to obtain a solution with finite boundary values (6.11), and then taking the limit as $\delta \rightarrow 0$ to obtain the blow-up solution. Moreover the relevant constructions may be performed in a neighborhood of each component, so that this process of letting $\varepsilon \rightarrow 0$ first, and then taking the limit $\delta \rightarrow 0$, may be carried out to obtain the desired result.

We now give an example to show that the nice asymptotics described in Theorems 6.3 and 6.5 can fail if $b=\frac{l+1}{2}$. In particular, we will exhibit a solution of the generalized Jang equation which sticks to the cylinder.

Example 6.6. Let us consider the exterior region of the Schwarzschild spacetime with metric

$$
-\left(1-\frac{2 m}{r}\right) d t^{2}+\left(1-\frac{2 m}{r}\right)^{-1} d r^{2}+r^{2} d \sigma^{2},
$$

where $d \sigma^{2}$ is the round metric on $\mathbb{S}^{2}$. Let $t=f(r)$ be a radial graph, with induced metric

$$
g=\left(\left(1-\frac{2 m}{r}\right)^{-1}-\left(1-\frac{2 m}{r}\right) f^{\prime 2}\right) d r^{2}+r^{2} d \sigma^{2} .
$$

As is calculated in [5], the second fundamental form of the graph is given by

$$
k_{i j}=\frac{\phi \nabla_{i j} f+\phi_{i} f_{j}+\phi_{j} f_{i}}{\sqrt{1+\phi^{2}|\nabla f|^{2}}}
$$

where $\phi=\sqrt{1-\frac{2 m}{r}}$ and the covariant derivatives are calculated with respect to the metric $g$. Thus $\left(M=\mathbb{R}^{3}-B_{2 m}(0), g, k\right)$ forms an initial data set for which the graph $t=f(r)$ is a solution of the generalized Jang equation. We choose a solution $t=f(r)$ such that the function $f$ is smooth up to the boundary $r=2 m$. In particular, $f(2 m)<\infty$ and we may consider this as an example of a Jang graph 'sticking' to the cylinder. For such an $f$, we note that

$$
g_{11}=\left(1-\frac{2 m}{r}\right)^{-1}-\left(1-\frac{2 m}{r}\right) f^{\prime 2} \sim\left(1-\frac{2 m}{r}\right)^{-1} .
$$

Therefore the distance to the boundary is given by

$$
\tau=\int_{2 m}^{r} \sqrt{g_{11}} \sim \sqrt{1-\frac{2 m}{r}} .
$$


This implies that $\phi \sim \tau$, or rather $b=1$. We now compute the null expansion of the coordinate spheres with respect to the initial data metric $g$. A standard formula [5] yields

$$
H_{S_{r}}=\frac{2 \sqrt{g^{11}}}{r} \sim \sqrt{1-\frac{2 m}{r}}
$$

and the trace of the initial data $k$ over the coordinate spheres is given by

$$
\operatorname{Tr}_{S_{r}} k=-\frac{\phi \gamma^{i j} \Gamma_{i j}^{1} f^{\prime}}{\sqrt{1+\phi^{2}|\nabla f|^{2}}}=\frac{2}{r} \frac{\phi g^{11} f^{\prime}}{\sqrt{1+\phi^{2} g^{11} f^{\prime 2}}} \sim\left(1-\frac{2 m}{r}\right)^{\frac{3}{2}} .
$$

It follows that

$$
\theta_{ \pm}=H_{S_{r}} \pm \operatorname{Tr}_{S_{r}} k \sim \sqrt{1-\frac{2 m}{r}}
$$

In terms of the notation used above, we have $\theta_{ \pm} \sim \tau$, and in particular, $l=1$.

\section{REFERENCES}

1. L. Andersson, J. Metzger, The area of horizons and the trapped region, Comm. Math. Phys., 290 (2009), no. 3, 941-972.

2. L. Andersson, J. Metzger, Curvature estimates for stable marginally trapped surfaces, J. Differential Geom., 84 (2010), 231-265.

3. L. Andersson, M. Eichmair, J. Metzger, Jang's equation and its applications to marginally trapped surfaces, Contemporary Mathematics, Complex Analysis and Dynamical Systems IV: Part 2. General Relativity, Geometry, and PDE (2011), 13-46.

4. H. Bray, Proof of the Riemannian Penrose conjecture using the positive mass theorem, J. Differential Geom., 59 (2001), 177-267.

5. H. Bray, M. Khuri, A Jang equation approach to the Penrose inequality, Discrete Contin. Dyn. Syst., 27 (2010), 741-766. arXiv:0910.4785

6. H. Bray, M. Khuri, P.D.E's which imply the Penrose conjecture, Asian J. Math., 15 (2011), no. 4, 557-610. arXiv:0905.2622

7. M. Disconzi, M. Khuri, On the Penrose inequality for charged black holes, Class. Quantum Grav., 29 (2012), 245019. arXiv:1207.5484

8. M. Eichmair, J. Metzger, Jenkins-Serrin type results for the Jang equation, preprint, 2012, arXiv:1205.4301.

9. G. Galloway, Rigidity of marginally trapped surfaces and the topology of black holes, Commun. Anal. Geom., 16 (2008), 217-229.

10. G. Galloway, R. Schoen, A generalization of Hawking's black hole topology theorem to higher dimensions, Comm. Math. Phys., 266 (2006), no. 2, 571-576.

11. D. Gilbarg, N. Trudinger, Elliptic Partial Differential Equations of Second Order, Springer-Verlag, New York, 1998.

12. D. Hoffman, J. Spruck, Sobolev and isoperimetric inequalities for riemannian submanifolds, Communications on Pure and Applied Mathematics, 27 (1974), 715-727.

13. G. Huisken, T. Ilmanen, The inverse mean curvature flow and the Riemannian Penrose inequality, J. Differential Geom., 59 (2001), 353-437.

14. P.-S. Jang, On the positivity of energy in General Relativity, J. Math. Phys., 19 (1978), 1152-1155.

15. M. Khuri, G. Weinstein, Rigidity in the positive mass theorem with charge, J. Math. Phys., 54 (2013), 092501. arXiv:1307.5499

16. E. Malec, N. Ó Murchadha, The Jang equation, apparent horizons, and the Penrose inequality, Class. Q. Grav., 21 (2004), 5777-5787.

17. J. Metzger, Blowup of Jang's equation at outermost marginally trapped surfaces, Comm. Math. Phys., 294 (2010), no. 1, 61-72.

18. R. Schoen, S.-T. Yau, On the positive mass conjecture in general relativity, Comm. Math. Phys., 65 (1979), no. 1, 45-76.

19. R. Schoen, S.-T. Yau, Proof of the positive mass theorem II, Comm. Math. Phys., 79 (1981), no. 2, 231-260.

20. E. Witten, A new proof of the positive energy theorem, Comm. Math. Phys., 80 (1981), no. 3, 381-402. 
Department of Mathematics, University of Notre Dame, Notre Dame, IN 46556

E-mail address: qhan@nd.edu

Beijing International Center for Mathematical Research, Peking University, Beijing, 100871, China

E-mail address: qhan@math.pku.edu.cn

Department of Mathematics, Stony Brook University, Stony Brook, Ny 11794

E-mail address: khuri@math.sunysb.edu 